\title{
Comparative Analysis of Fatty Acid Desaturases in Cyanobacterial Genomes
}

\author{
Xiaoyuan Chi, 1, 2, 3 Qingli Yang, 1, 2,3 Fangqing Zhao, ${ }^{1,2}$ Song Qin, ${ }^{1}$ Yu Yang, ${ }^{1,2}$ \\ Junjun Shen, ${ }^{1,2}$ and Hanzhi Lin ${ }^{1,2}$ \\ ${ }^{1}$ Key Laboratory of Experimental Marine Biology, Institute of Oceanology, Chinese Academy of Sciences, \\ Qingdao 266071, China \\ ${ }^{2}$ Graduate University, Chinese Academy of Sciences, Beijing 100039, China \\ ${ }^{3}$ Shandong Peanut Research Institute, Qingdao 266100, China \\ Correspondence should be addressed to Song Qin, sqin@ms.qdio.ac.cn
}

Received 23 August 2007; Revised 17 March 2008; Accepted 4 September 2008

Recommended by John Parkinson

\begin{abstract}
Fatty acid desaturases are enzymes that introduce double bonds into the hydrocarbon chains of fatty acids. The fatty acid desaturases from 37 cyanobacterial genomes were identified and classified based upon their conserved histidine-rich motifs and phylogenetic analysis, which help to determine the amounts and distributions of desaturases in cyanobacterial species. The filamentous or $\mathrm{N}_{2}$-fixing cyanobacteria usually possess more types of fatty acid desaturases than that of unicellular species. The pathway of acyl-lipid desaturation for unicellular marine cyanobacteria Synechococcus and Prochlorococcus differs from that of other cyanobacteria, indicating different phylogenetic histories of the two genera from other cyanobacteria isolated from freshwater, soil, or symbiont. Strain Gloeobacter violaceus PCC 7421 was isolated from calcareous rock and lacks thylakoid membranes. The types and amounts of desaturases of this strain are distinct to those of other cyanobacteria, reflecting the earliest divergence of it from the cyanobacterial line. Three thermophilic unicellular strains, Thermosynechococcus elongatus BP-1 and two Synechococcus Yellowstone species, lack highly unsaturated fatty acids in lipids and contain only one $\Delta 9$ desaturase in contrast with mesophilic strains, which is probably due to their thermic habitats. Thus, the amounts and types of fatty acid desaturases are various among different cyanobacterial species, which may result from the adaption to environments in evolution.
\end{abstract}

Copyright () 2008 Xiaoyuan Chi et al. This is an open access article distributed under the Creative Commons Attribution License, which permits unrestricted use, distribution, and reproduction in any medium, provided the original work is properly cited.

\section{Introduction}

In living organisms, the regulation of membrane fluidity is necessary for the proper function of biological membranes, which is important in the tolerance and acclimatization to environmental stresses such as heat, cold, desiccation, salinity, nitrogen starvation, photooxidation, anaerobiosis, and osmosis, and so forth. Unsaturated fatty acids are essential constituents of polar glycerolipids in biological membranes and the unsaturation level of membrane lipids is important in controlling the fluidity of membranes [1]. Fatty acid desaturases are enzymes that introduce double bonds into the hydrocarbon chains of fatty acids to produce unsaturated and polyunsaturated fatty acids [2], thus these enzymes play an important role during the process of environmental adaptation.
Cyanobacteria, prokaryotes capable of carrying out a plant-like oxygenic photosynthesis, represent one of the oldest known bacterial lineages, with fossil evidence suggesting an appearance around 3-3.5 billion years ago [3]. Cyanobacteria comprise over 1600 species with various morphologies and species-specific characteristics such as cell movement, cell differentiation, and nitrogen fixation [4]. Extant cyanobacteria can be found in virtually all ecosystem habitats on Earth, ranging from the freshwater lakes and rivers through to the oceans, and also in hot springs and deserts, ranging from the hottest to the cold dry valleys of Antarctica [3].

Polyunsaturated membrane lipids play important roles in the growth, respiration, and photosynthesis of cyanobacteria. It is well documented that the content of polyunsaturated fatty acids in membrane lipids of cyanobacteria can be 

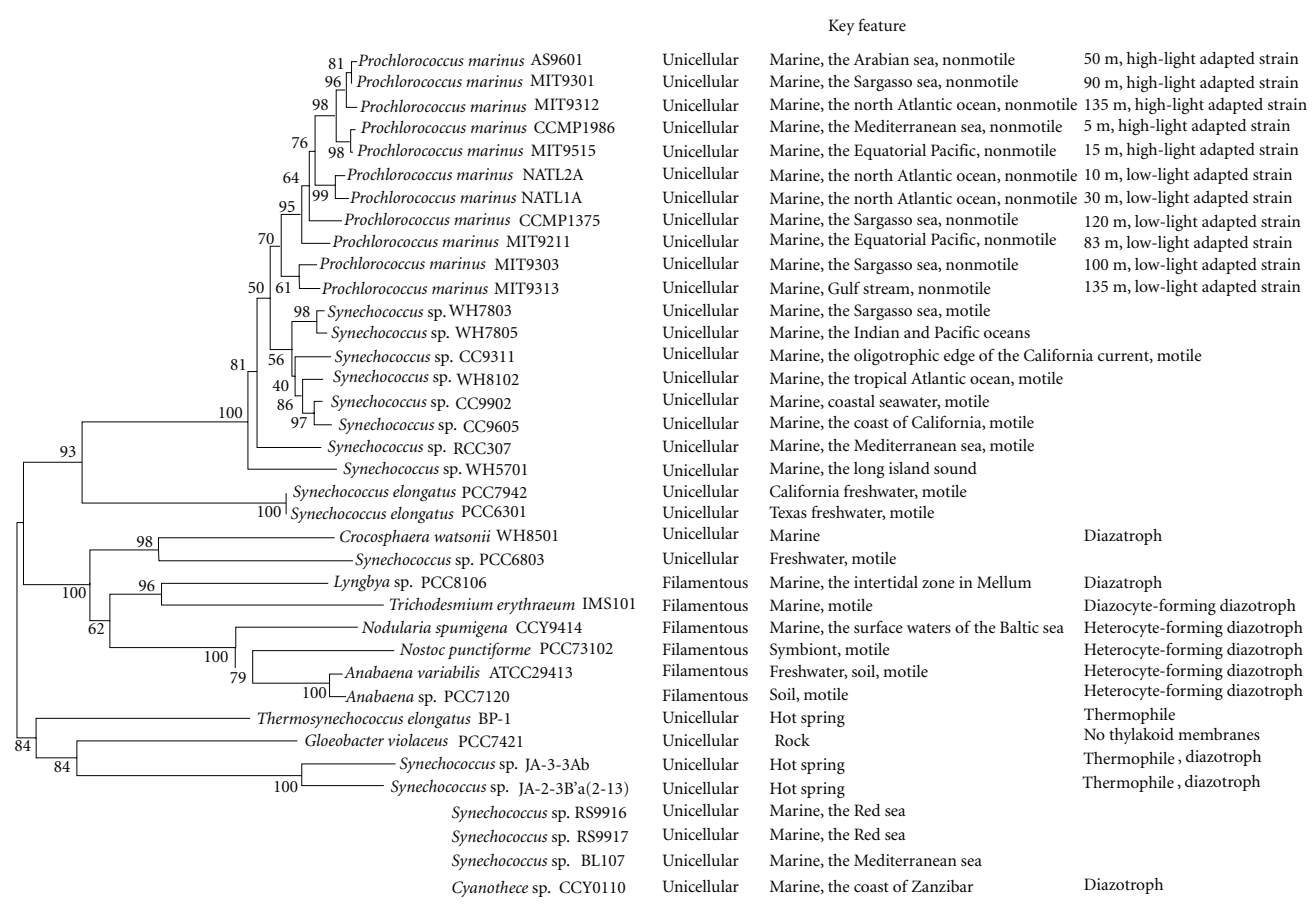

$\begin{array}{lcc}\text { Acronym } & \text { Status } & \text { Genome size } \\ \text { Pm9601 } & \text { Finished } & 1669886 \\ \text { Pm9301 } & \text { Finished } & 1641879 \\ \text { Pm9312 } & \text { Finished } & 1709204 \\ \text { Pm1986 } & \text { Finished } & 1657990 \\ \text { Pm9515 } & \text { Finished } & 1704176 \\ \text { Pm2A } & \text { Finished } & 1842899 \\ \text { Pm1A } & \text { Finished } & 1864731 \\ \text { Pm1375 } & \text { Finished } & 1751080 \\ \text { Pm9211 } & \text { Draft } & 1839003 \\ \text { Pm9303 } & \text { Finished } & 2682675 \\ \text { Pm9313 } & \text { Finished } & 2410873 \\ \text { SWH7803 } & \text { Finished } & 2366980 \\ \text { SWH7805 } & \text { Draft } & 2620367 \\ \text { S9311 } & \text { Finished } & 2606748 \\ \text { SWH8102 } & \text { Finished } & 2434428 \\ \text { S9902 } & \text { Finished } & 2234828 \\ \text { S9605 } & \text { Finished } & 2510659 \\ \text { S307 } & \text { Finished } & 2224914 \\ \text { SWH5701 } & \text { Draft } & 3043834 \\ \text { Se7942 } & \text { Finished } & 2742269 \\ \text { Se6301 } & \text { Finished } & 2696255 \\ \text { Cw8501 } & \text { Draft } & 6238156 \\ \text { S6803 } & \text { Finished } & 3947019 \\ \text { L8106 } & \text { Draft } & 7037511 \\ \text { Te101 } & \text { Finished } & 7750108 \\ \text { Ns9414 } & \text { Draft } & 5316258 \\ \text { Np73102 } & \text { Draft } & 9020037 \\ \text { Av29413 } & \text { Finished } & 7068601 \\ \text { A7120 } & \text { Finished } & 7211789 \\ \text { TeBP-1 } & \text { Finished } & 2593857 \\ \text { Gv7421 } & \text { Finished } & 4659019 \\ \text { SJA } & \text { Finished } & 2932766 \\ \text { SJB } & \text { Finished } & 3046680 \\ \text { S9916 } & \text { Draft } & 2664465 \\ \text { S9917 } & \text { Draft } & 2579542 \\ \text { S107 } & \text { Draft } & 2283377 \\ \text { C0110 } & \text { Draft } & 5880532 \\ & & \end{array}$

FIgURE 1: Phylogenetic tree of the sequenced cyanobacterial strains. A Neighbor-joining tree for 33 sequenced cyanobacteria constructed based on $16 \mathrm{~S}$ rRNA as was described in Section 2 and about 1300 positions were employed. To maximize the number of sites available for analysis, three partial sequences from Synechococcus sp. RS9917 (170 bp), Synechococcus sp. RS9916 (865 bp), and Synechococcus sp. BL107 (296 bp) were excluded. Moreover, no $16 \mathrm{~S}$ rRNA sequence was found in Cyanothece sp. CCY0110.

altered by changing the temperature [5-7]. The mechanism that regulates the fatty acid desaturation of membrane lipids in response to temperature has been demonstrated to be the result of the up- or downregulation of the expression of the desaturase genes [8]. Furthermore, it has been demonstrated that the position of double bonds in fatty acids is more influential on the fluidity of membrane lipids than the number of double bonds in fatty acids [9]. It is also found that the temperature of the phase transition dramatically decreased when the first and second double bonds are introduced into fatty acids, whereas the introduction of the third and fourth double bonds do not further lower the temperature of phase transition of membrane lipids [10].

Exposure of cyanobacteria to high PAR (photosynthetically active radiation) or UV radiation leads to photoinhibition of photosynthesis, thereby limiting the efficient fixation of light energy [11, 12]. In Synechocystis sp. PCC 6803, the replacement of all polyunsaturated fatty acids by a monounsaturated fatty acid suppressed the growth of the cells at low temperature, and it decreased the tolerance of the cells to photoinhibition of photosynthesis at low temperature by suppressing recovery of the photosystem II protein complex from photoinhibitory damage. However, the replacement of tri- and tetraunsaturated fatty acids by a diunsaturated fatty acid did not have such effects. These findings indicate that polyunsaturated fatty acids are important in protecting the photosynthetic machinery from photoinhibition at low temperatures [13]. Transformation of the cyanobacterium Synechococcus sp. PCC 7942 with the desA gene for a $\Delta 12$ desaturase has been reported to increase the unsaturation of membrane lipids and thereby enhance the tolerance of cyanobacterium to intense light. These findings demonstrate that the ability of membrane lipids to desaturate fatty acids is important for the photosynthetic organisms to be able to tolerate high-light stress by accelerating the synthesis of the $\mathrm{D}_{1}$ protein de novo [14].

Cyanobacteria have been classified into four groups in terms of the composition of fatty acids, the distribution of fatty acids at the $s n$ position of the glycerol moiety, and the position of double bonds in the fatty acids [15]. Strains in Group 1 (e.g., Prochlorothrix hollandica, Synechococcus sp. PCC 6301, Synechococcus sp. PCC 7942, Synechococcus elongatus, Thermosynechococcus elongates, and Thermosynechococcus vulcanus) introduce a double bond only at the $\Delta 9$ position of fatty acids at the $s n-1$ or $s n-2$ position of glycerolipids. Strains in Group 2 (e.g., Anabaena variabilis, Anabaena sp. PCC 7120, Synechococcus sp. PCC 7002, Nostoc punctiforme, and Nostoc sp. SO-36) introduce double bonds at the $\Delta 9, \Delta 12$, and $\Delta 15(\omega 3)$ positions of $\mathrm{C} 18$ acids at the $s n-1$ position, and at the $\Delta 9$ position of $\mathrm{C} 16$ acids at the $s n$ 2 position. Strains in Group 3 (e.g., Synechocystis sp. PCC 6714 and Spirulina platensis) can also introduce three double bonds, but these are at the $\Delta 6, \Delta 9$, and $\Delta 12$ positions of C18 acids at the $s n-1$ position. Strains in Group 4 (e.g., Synechocystis sp. PCC 6803 and Tolypothrix tenuis) introduce double bonds at the $\Delta 6, \Delta 9, \Delta 12$, and $\Delta 15$ ( $\omega 3)$ positions of $\mathrm{C} 18$ acids at the $s n-1$ position. The $\mathrm{C} 16$ acids at the $s n-2$ position are not desaturated in Groups 3 and 4. 
The entire genome sequence of a unicellular cyanobacterium Synechocystis sp. strain PCC 6803 was first described in 1996 [16]. To date, 37 cyanobacterial genomes have been sequenced (Figure 1). These genomes are those of the filamentous nitrogen-fixing cyanobacterium Anabaena sp. PCC 7120, the thermophilic strain Thermosynechococcus elongatus BP-1, the thylakoid-free strain Gloeobacter violaceus PCC 7421, the marine cyanobacterium Synechococcus sp. strain WH8102, the Prochlorococcus marinus strains SS120, MED4, MIT 9313, Synechococcus sp. CC9311, and others. These genome-sequencing projects undoubtedly bring a great convenience to obtain a comprehensive dataset of genes involved in unsaturated fatty acid biosynthesis in cyanobacteria. In this work, we identified all the putative fatty acid desaturases using bioinformatic tools and presented a genomic comparison of the fatty acid desaturases from 37 cyanobacterial genomes. The identification of novel desaturases and the reconstruction of the pathways for unsaturated fatty acid biosynthesis in cyanobacteria will guide the experimental analysis and provide clues in study of the relationship between the unsaturation level of membrane lipids and environmental adaptation in higher plants.

\section{Materials and Methods}

\subsection{Computational Search for Novel Fatty Acid Desaturase Genes}

The genomes of 37 cyanobacteria including genera Synechocystis, Synechococcus, Prochlorococcus, Anabaena, Nostoc, Trichodesmium, Gloeobacter, Crocosphaera, Cyanothece, and Lyngbya were downloaded from IMG database (http://img .jgi.doe.gov/cgi-bin/pub/main.cgi). The dataset comprised of well-characterized fatty acid desaturases from Synechocystis PCC 6803 (NP_442430, NP_441489, NP_441622, NP_441824), Nostoc sp. SO-36 (CAF18426), Synechococcus sp. PCC 7002 (AAB61353, AAF21445, AAB61352), Arthrospira platensis (CAA05166, Q54794, CAA60573), Synechococcus vulcanus (AAD00699), Synechococcus s elongatus sp. PCC 6301 (YP_172259), Synechococcus elongatus sp. PCC 7942 (YP_401578), Phaeodactylum tricorutum (AAW70158, AY082393, AAO23565, AY165023), Chlamydomonas reinhardtii (AB007640, ABL09485, EDP04777), and Chlorella vulgaris (AB075526, AB075527) was used to construct a query protein set. Each protein in this query dataset was used to search the potential novel sequences in 37 cyanobacterial species with whole genome sequences available, by using the BLASTP and TBLASTN programs, with $E$-value $<1 e-10$. The searches were repeated until no novel sequences were detected at the $e$ value threshold used. The putative desaturase genes across 37 genomes were summarized in Table 1. The other amino acid sequences beyond the 37 cyanobacterial species were retrieved from NCBI (http://www.ncbi.nlm.nih.gov/). The accession number of these sequences and the names of corresponding cyanobacteria, eukaryotic algae, higher plants, fungi, and animals were indicated in Table 2.

\subsection{Multiple Sequence Alignment and Phylogenetic Analysis}

Sequence alignments were generated using Clustal $\mathrm{W}$ program [17]. The SMART (http://smart.embl-heidelberg.de/) and PFAM (http://pfam.sanger.ac.uk/) databases were used to search the conserved domains of the putative desaturase enzymes. The conserved amino acid residues of different conserved domains were manually identified using the BioEdit sequence editor. The final alignment was further refined after excluding the poorly conserved regions at the protein ends, and consisted of sequences spanning the conserved domains. The neighbor-joining (NJ) and minimum-evolution (ME) methods in MEGA4 [18] were used to construct the phylogenetic tree. To maximize the number of sites available for analysis, two partial sequences from Synechococcus sp. WH 7805 (ZP_01124768, 174 aa) and Nodularia spumigena CCY9414 (ZP_01629726, 196 aa) were excluded. Bootstrap with 1000 replicates was used to establish the confidence limit of the tree branches.

\section{Results and Discussions}

\subsection{The Conserved Motifs}

Using BlastP and TBlastN programs with the query sequences to search the 37 genomes of cyanobacteria, 193 protein sequences were identified including fatty acid desaturase, fatty acid dehydrogenase, hypothetical protein, $\beta$ carotene ketolase, $\beta$-carotene hydroxylase, and hydrocarbon oxygenase. PFAM and SMART domain analyses could not distinguish fatty acid desaturase from fatty acid dehydrogenase, $\beta$-carotene ketolase, $\beta$-carotene hydroxylase, or hydrocarbon oxygenase. Moreover, most of the protein sequences which were originally annotated as fatty acid desaturase were not classified into $\Delta 9, \Delta 12, \Delta 15$, or $\Delta 6$ desaturase categories. To facilitate the classification of different types of desaturases, the conserved motifs of different enzymes were identified by multiple sequence alignments with Clustal W.

There were three typical histidine-rich motifs existed in all the proteins similar to proven cyanobacterial fatty acid desaturases (Table 3). Moreover, there were different conserved residues in the same histidine-boxes of different kinds of proteins, suggesting that these proteins might have acquired different functions from a common ancestor during the evolution. According to the different conserved residues of three histidine-motifs and phylogenetic profile, $16 \beta$-carotene ketolases, $36 \beta$-carotene hydroxylases, and 8 hydrocarbon oxygenases (MocD, a rhizopine oxygenase for the conversion of 3-O-MSI to SI)) were identified from the 37 cyanobacterial genomes (Figures 2, 4, and 5).

\subsection{Discovery of Candidate Genes for $\Delta 9$ Desaturases}

To elucidate the phylogenetic relationships among different membrane desaturases, genes from cyanobacteria, eukaryotic algae, higher plants, fungi, invertebrates, and vertebrates 
TABLE 1: Lists of putative desaturase genes from thirty seven cyanobacterial genomes.

\begin{tabular}{|c|c|c|c|c|c|}
\hline Species & Locus tag & Accession & DNA coordinates & Length & Proposed function \\
\hline \multirow{6}{*}{ Anabaena sp. PCC 7120} & all4991 & NP_489031 & $5963080 \cdots 5963937$ & 857 & d9 \\
\hline & all1599 & NP_485639 & $1879629 \cdots 1880447$ & 818 & d9 \\
\hline & all1598 & NP_485638 & $1878346 \cdots 1879398$ & 1052 & $\mathrm{~d} 12$ \\
\hline & all1597 & NP_485637 & $1876897 \cdots 1877976$ & 1079 & $\mathrm{~d} 15$ \\
\hline & alr3189 & NP_487229 & $3858986 \cdots 3859762$ & 776 & crtW \\
\hline & alr4009 & NP_488049 & $4829483 \cdots 4830322$ & 839 & crtR \\
\hline \multirow{7}{*}{$\begin{array}{l}\text { Anabaena variabilis ATCC } \\
29413\end{array}$} & Ava_2277 & YP_322790 & $2832413 \cdots 2833270$ & 857 & d9 \\
\hline & Ava_4212 & YP_324706 & $5282348 \cdots 5283166$ & 818 & d9 \\
\hline & Ava_4211 & YP_324705 & $5281066 \cdots 5282118$ & 1052 & $\mathrm{~d} 12$ \\
\hline & Ava_4210 & YP_324704 & $5279614 \cdots 5280693$ & 1079 & $\mathrm{~d} 15$ \\
\hline & Ava_2048 & YP_322565 & $2535646 \cdots 2536410$ & 764 & $\operatorname{crtW}$ \\
\hline & Ava_3888 & YP_324388 & $4842189 \cdots 4842965$ & 776 & $\operatorname{crtW}$ \\
\hline & Ava_1693 & YP_322210 & $2121129 \cdots 2122049$ & 920 & $\operatorname{crtR}$ \\
\hline \multirow{6}{*}{$\begin{array}{l}\text { Crocosphaera watsonii WH } \\
8501\end{array}$} & CwatDRAFT_1377 & ZP_00518170 & $3068 \cdots 3892$ & 824 & d9 \\
\hline & CwatDRAFT_3226 & ZP_00516843 & $22017 \cdots 23066$ & 1049 & d12 \\
\hline & CwatDRAFT_5150 & ZP_00515010 & $150888 \cdots 151982$ & 1049 & d 12 \\
\hline & CwatDRAFT_3625 & ZP_00516181 & $10760 \cdots 11809$ & 1049 & $\mathrm{~d} 15$ \\
\hline & CwatDRAFT_1857 & ZP_00517700 & $1398 \cdots 2231$ & 834 & hypothetical protein \\
\hline & CwatDRAFT_5424 & ZP_00514501 & $315629 \cdots 316522$ & 893 & crtR \\
\hline \multirow{11}{*}{$\begin{array}{l}\text { Gloeobacter violaceus strain } \\
\text { PCC } 7421\end{array}$} & gvip390 & NP_925812 & $3057506 \cdots 3058357$ & 851 & d9 \\
\hline & gvip170 & NP_924181 & $1312274 \cdots 1313095$ & 822 & d 9 \\
\hline & gll1946 & NP_924892 & $2071551 \cdots 2072504$ & 953 & d 9 \\
\hline & gll1947 & NP_924893 & $2072509 \cdots 2073507$ & 998 & d 9 \\
\hline & gll1938 & NP_924884 & $2060880 \cdots 2061839$ & 959 & d9 \\
\hline & gll1940 & NP_924886 & $2063884 \cdots 2064876$ & 992 & d 9 \\
\hline & gvip364 & NP_925569 & $2779580 \cdots 2780638$ & 1058 & $\mathrm{~d} 12$ \\
\hline & gvip506 & NP_926681 & $3944843 \cdots 3945910$ & 1058 & $\mathrm{~d} 12$ \\
\hline & gll0171 & NP_923117 & $161268 \cdots 162440$ & 1173 & hypothetical protein \\
\hline & gll2501 & NP_925447 & $2660474 \cdots 2661475$ & 1001 & $\operatorname{mocD}$ \\
\hline & gvip239 & NP_924674 & $1833712 \cdots 1834485$ & 773 & crtW \\
\hline \multirow{11}{*}{$\begin{array}{l}\text { Nostoc punctiforme ATCC } \\
\text { 29133(PCC 73102) }\end{array}$} & Npun02000467 & ZP_00345918 & $175651 \cdots 176532$ & 881 & d9 \\
\hline & Npun02005010 & ZP_00108582 & $41108 \cdots 41929$ & 821 & d9 \\
\hline & Npun02005011 & ZP_00108583 & $42265 \cdots 43326$ & 1061 & $\mathrm{~d} 12$ \\
\hline & Npun02005012 & ZP_00108584 & $43524 \cdots 44603$ & 1080 & $\mathrm{~d} 15$ \\
\hline & Npun02001904 & ZP_00345765 & $63255 \cdots 64310$ & 1056 & hypothetical protein \\
\hline & Npun02001905 & ZP_00110890 & $64537 \cdots 65574$ & 1038 & hypothetical protein \\
\hline & Npun02002344 & ZP_00110549 & $77763 \cdots 78863$ & 1101 & hypothetical protein \\
\hline & Npun02003462 & ZP_00109371 & $76020 \cdots 76964$ & 945 & $\operatorname{mocD}$ \\
\hline & Npun02000865 & ZP_00345866 & $139810 \cdots 140571$ & 762 & crtW \\
\hline & Npun02001326 & ZP_00111258 & $55604 \cdots 56392$ & 788 & crtW \\
\hline & Npun02006805 & ZP_00106832 & $23657 \cdots 24556$ & 899 & crtR \\
\hline \multirow{3}{*}{$\begin{array}{l}\text { Prochlorococcus marinus str. } \\
\text { NATL1A }\end{array}$} & NATL1_21421 & YP_001015962 & $1799954 \cdots 1800733$ & 780 & d9 \\
\hline & NATL1_10821 & YP_001014905 & 992775 . . 993992 & 1218 & $\mathrm{~d} 12$ \\
\hline & NATL1_03151 & YP_001014144 & $291853 \cdots 292884$ & 1032 & $\operatorname{crtR}$ \\
\hline \multirow{3}{*}{$\begin{array}{l}\text { Prochlorococcus marinus } \\
\text { strain NATL2A }\end{array}$} & PMN2A_1271 & YP_292464 & $1227545 \cdots 1228474$ & 929 & d9 \\
\hline & PMN2A_0393 & YP_291588 & $388657 \cdots 389874$ & 1217 & $\mathrm{~d} 12$ \\
\hline & PMN2A_1603 & YP_292794 & $1566557 \cdots 1567588$ & 1031 & crtR \\
\hline
\end{tabular}


TABle 1: Continued.

\begin{tabular}{|c|c|c|c|c|c|}
\hline Species & Locus tag & Accession & DNA coordinates & Length & Proposed function \\
\hline \multirow{4}{*}{$\begin{array}{l}\text { Prochlorococcus marinus } \\
\text { MIT } 9211\end{array}$} & P9211_09157 & ZP_01006363 & $1417821 \cdots 1418765$ & 944 & $\mathrm{~d} 9$ \\
\hline & P9211_05577 & ZP_01005647 & $779723 \cdots 780334$ & 611 & $\mathrm{~d} 12$ \\
\hline & P9211_05582 & ZP_01005648 & $780304 \cdots 780729$ & 425 & $\mathrm{~d} 12$ \\
\hline & P9211_07547 & ZP_01006041 & $1108444 \cdots 1109469$ & 1015 & $\mathrm{crtR}$ \\
\hline \multirow{4}{*}{$\begin{array}{l}\text { Prochlorococcus marinus str. } \\
\text { MIT } 9301\end{array}$} & P9301_18621 & YP_001092086 & $1588713 \cdots 1589651$ & 939 & d9 \\
\hline & P9301_15761 & YP_001091800 & $1328773 \cdots 1329939$ & 1167 & $\mathrm{~d} 12$ \\
\hline & P9301_15721 & YP_001091796 & $1326076 \cdots 1327182$ & 1107 & $\mathrm{~d} 12$ \\
\hline & P9301_02581 & YP_001090482 & $239249 \cdots 239974$ & 726 & $\operatorname{crtR}$ \\
\hline \multirow{5}{*}{$\begin{array}{l}\text { Prochlorococcus marinus str. } \\
\text { MIT } 9303\end{array}$} & P9303_28951 & YP_001018890 & $2560285 \cdots 2561250$ & 966 & d9 \\
\hline & P9303_28931 & YP_001018888 & $2558615 \cdots 2559535$ & 921 & d9 \\
\hline & P9303_14121 & YP_001017424 & $1208715 \cdots 1209800$ & 1086 & $\mathrm{~d} 12$ \\
\hline & P9303_21081 & YP_001018108 & $1869188 \cdots 1870330$ & 1143 & $\mathrm{~d} 12$ \\
\hline & P9303_24321 & YP_001018428 & $2137288 \cdots 2138328$ & 1041 & crtR \\
\hline \multirow{4}{*}{$\begin{array}{l}\text { Prochlorococcus marinus str. } \\
\text { MIT } 9312\end{array}$} & PMT9312_1764 & YP_398261 & $1656076 \cdots 1657014$ & 938 & d9 \\
\hline & PMT9312_1476 & YP_397972 & $1385670 \cdots 1386845$ & 1175 & $\mathrm{~d} 12$ \\
\hline & PMT9312_1473 & YP_397969 & $1382796 \cdots 1383902$ & 1106 & $\mathrm{~d} 12$ \\
\hline & РMT9312_0238 & YP_396735 & $229042 \cdots 229842$ & 800 & $\operatorname{crtR}$ \\
\hline \multirow{5}{*}{$\begin{array}{l}\text { Prochlorococcus marinus str. } \\
\text { MIT } 9313\end{array}$} & PMT2172 & NP_895996 & $2299082 \cdots 2300002$ & 920 & d9 \\
\hline & PMT2174 & NP_895998 & $2300938 \cdots 2301717$ & 779 & d9 \\
\hline & РMT0249 & NP_894082 & $278544 \cdots 279683$ & 1139 & $\mathrm{~d} 12$ \\
\hline & PMT0797 & NP_894629 & $872385 \cdots 873470$ & 1085 & $\mathrm{~d} 12$ \\
\hline & PMT1816 & NP_895643 & $1920323 \cdots 1921363$ & 1040 & $\operatorname{crtR}$ \\
\hline \multirow{4}{*}{$\begin{array}{l}\text { Prochlorococcus marinus str. } \\
\text { AS9601 }\end{array}$} & A9601_18811 & YP_001010271 & $1616719 \cdots 1617657$ & 939 & d9 \\
\hline & A9601_15921 & YP_001009982 & $1355480 \cdots 1356514$ & 1035 & $\mathrm{~d} 12$ \\
\hline & A9601_15871 & YP_001009977 & $1352826 \cdots 1353932$ & 1107 & $\mathrm{~d} 12$ \\
\hline & A9601_02571 & YP_001008652 & $238284 \cdots 239117$ & 834 & crtR \\
\hline \multirow{4}{*}{$\begin{array}{l}\text { Prochlorococcus marinus str. } \\
\text { MIT } 9515\end{array}$} & P9515_18621 & YP_001012176 & $1650943 \cdots 1651929$ & 987 & d9 \\
\hline & P9515_15601 & YP_001011874 & $1376566 \cdots 1377693$ & 1128 & $\mathrm{~d} 12$ \\
\hline & P9515_15521 & YP_001011866 & $1371646 \cdots 1372752$ & 1107 & $\mathrm{~d} 12$ \\
\hline & P9515_02681 & YP_001010584 & $247534 \cdots 248433$ & 900 & $\operatorname{crtR}$ \\
\hline \multirow{4}{*}{$\begin{array}{l}\text { Prochlorococcus marinus } \\
\text { subsp. marinus str. } \\
\text { CCMP1375 (SS120) }\end{array}$} & Pro1833 & NP_876224 & $1690865 \cdots 1691797$ & 932 & d9 \\
\hline & Pro1208 & NP_875600 & $1116904 \cdots 1118016$ & 1112 & $\mathrm{~d} 12$ \\
\hline & Pro1214 & NP_875606 & $1121144 \cdots 1122250$ & 1106 & $\mathrm{~d} 12$ \\
\hline & Pro0266 & NP_874660 & $261189 \cdots 262223$ & 1034 & $\operatorname{crtR}$ \\
\hline \multirow{4}{*}{$\begin{array}{l}\text { Prochlorococcus marinus } \\
\text { subsp. marinus str. } \\
\text { CCMP1986 (MED4) }\end{array}$} & PMM1672 & NP_893789 & $1604745 \cdots 1605731$ & 986 & d9 \\
\hline & PMM1382 & NP_893499 & $1331162 \cdots 1332340$ & 1178 & $\mathrm{~d} 12$ \\
\hline & PMM1378 & NP_893495 & $1325388 \cdots 1326494$ & 1106 & $\mathrm{~d} 12$ \\
\hline & PMM0236 & 1 & $228281 \cdots 229270$ & 989 & $\operatorname{crtR}$ \\
\hline \multirow{3}{*}{$\begin{array}{l}\text { Synechococcus elongatus } \\
\text { strain PCC } 7942\end{array}$} & Synpcc7942_2561 & YP_401578 & $2639146 \cdots 2639982$ & 836 & d9 \\
\hline & Synpcc7942_1713 & YP_400730 & $1781317 \cdots 1782219$ & 902 & $\operatorname{mocD}$ \\
\hline & Synpcc7942_2439 & YP_401456 & $2514276 \cdots 2515271$ & 995 & crtR \\
\hline \multirow{3}{*}{$\begin{array}{l}\text { Synechococcus elongatus } \\
\text { strain PCC } 6301\end{array}$} & syc1549_d & YP_172259 & $1676804 \cdots 1677640$ & 837 & d9 \\
\hline & Syc2378_c & YP_173088 & $2534831 \cdots 2535691$ & 861 & $\operatorname{mocD}$ \\
\hline & syc1667_c & YP_172377 & $1801757 \cdots 1802752$ & 996 & crtR \\
\hline \multirow{5}{*}{ Synechococcus sp. BL107 } & BL107_07284 & ZP_01469203 & $490784 \cdots 491566$ & 782 & d9 \\
\hline & BL107_07289 & ZP_01469204 & $491936 \cdots 492721$ & 785 & d9 \\
\hline & BL107_06084 & ZP_01468963 & $247334 \cdots 248356$ & 1022 & $\mathrm{~d} 12$ \\
\hline & BL107_14110 & ZP_01468055 & $331111 \cdots 331884$ & 773 & crtW \\
\hline & BL107_08054 & ZP_01469357 & $636707 \cdots 637738$ & 1031 & $\operatorname{crtR}$ \\
\hline
\end{tabular}


Table 1: Continued.

\begin{tabular}{|c|c|c|c|c|c|}
\hline Species & Locus tag & Accession & DNA coordinates & Length & Proposed function \\
\hline \multirow{5}{*}{ Synechococcus sp. CC9311 } & sync_2793 & YP_731981 & $2458778 \cdots 2459710$ & 932 & d9 \\
\hline & sync_2791 & YP_731979 & $2457075 \cdots 2457986$ & 911 & d9 \\
\hline & sync_0336 & YP_729569 & $344430 \cdots 345449$ & 1019 & $\operatorname{crtR}$ \\
\hline & sync_0396 & YP_729627 & $408306 \cdots 409505$ & 1199 & $\mathrm{~d} 12$ \\
\hline & sync_1804 & YP_731008 & $1621108 \cdots 1621869$ & 761 & crtW \\
\hline \multirow{3}{*}{ Synechococcus sp. CC9605 } & Syncc9605_2541 & YP_382824 & $2358792 \cdots 2359703$ & 911 & d9 \\
\hline & Syncc9605_1972 & YP_382268 & $1793076 \cdots 1794221$ & 1145 & $\mathrm{~d} 12$ \\
\hline & Syncc9605_0286 & YP_380617 & $292821 \cdots 293870$ & 1049 & crtR \\
\hline \multirow{5}{*}{ Synechococcus sp. CC9902 } & Syncc9902_2191 & YP_378192 & $2099771 \cdots 2100673$ & 902 & d9 \\
\hline & Syncc9902_2192 & YP_378193 & $2100902 \cdots 2101825$ & 923 & d9 \\
\hline & Syncc9902_0141 & YP_376159 & $149723 \cdots 150724$ & 1001 & $\mathrm{~d} 12$ \\
\hline & Syncc9902_0972 & YP_376982 & $954015 \cdots 954788$ & 773 & crtW \\
\hline & Syncc9902_2058 & YP_378059 & $1964618 \cdots 1965730$ & 1112 & $\operatorname{crtR}$ \\
\hline \multirow{3}{*}{$\begin{array}{l}\text { Synechococcus sp. } \\
\text { JA-2-3B' a (2-13) }\end{array}$} & CYB_0861 & YP_477105 & $894187 \cdots 895071$ & 884 & d9 \\
\hline & CYB_2914 & YP_479096 & $3011594 \cdots 3012520$ & 926 & $\operatorname{mocD}$ \\
\hline & CYB_0102 & YP_476366 & $118335 \cdots 119306$ & 971 & crtR \\
\hline \multirow{2}{*}{ Synechococcus sp. JA-3-3Ab } & CYA_2349 & YP_475739 & $2357019 \cdots 2357912$ & 893 & d9 \\
\hline & CYA_1931 & YP_475340 & $1944066 \cdots 1945040$ & 974 & crtR \\
\hline \multirow{5}{*}{ Synechococcus sp. RCC307 } & SynRCC307_2395 & YP_001228651 & $2091372 \cdots 2092274$ & 903 & d9 \\
\hline & SynRCC307_2393 & YP_001228649 & $2089667 \cdots 2090581$ & 915 & d9 \\
\hline & SynRCC307_1757 & YP_001228013 & $1538507 \cdots 1539562$ & 1056 & $\mathrm{~d} 12$ \\
\hline & SynRCC307_1993 & YP_001228249 & $1729342 \cdots 1730103$ & 762 & crtW \\
\hline & SynRCC307_2209 & YP_001228465 & $1915148 \cdots 1916167$ & 1020 & $\operatorname{crtR}$ \\
\hline \multirow{3}{*}{ Synechococcus sp. RS9916 } & RS9916_36767 & ZP_01471384 & $1050409 \cdots 1051341$ & 932 & d9 \\
\hline & RS9916_36757 & ZP_01471382 & $1048603 \cdots 1049568$ & 965 & d9 \\
\hline & RS9916_39311 & ZP_01472905 & $116650 \cdots 117675$ & 1025 & $\operatorname{crtR}$ \\
\hline \multirow{5}{*}{ Synechococcus sp. RS9917 } & RS9917_06370 & ZP_01079314 & $447782 \cdots 448705$ & 923 & d9 \\
\hline & RS9917_06360 & ZP_01079312 & $446060 \cdots 446992$ & 932 & d9 \\
\hline & RS9917_03333 & ZP_01080849 & $99968 \cdots 101047$ & 1079 & $\mathrm{~d} 12$ \\
\hline & RS9917_00687 & ZP_01080541 & $64826 \cdots 65563$ & 737 & crtW \\
\hline & RS9917_03663 & ZP_01080915 & $166940 \cdots 167902$ & 962 & $\operatorname{crtR}$ \\
\hline \multirow{8}{*}{ Synechococcus sp. WH 5701} & WH5701_02025 & ZP_01084898 & $299319 \cdots 300257$ & 787 & d9 \\
\hline & WH5701_02015 & ZP_01084896 & $297579 \cdots 298532$ & 953 & d9 \\
\hline & WH5701_14646 & ZP_01083974 & $104382 \cdots 105539$ & 1157 & $\mathrm{~d} 12$ \\
\hline & WH5701_16535 & ZP_01086617 & $164 \cdots 1186$ & 1022 & $\mathrm{~d} 12$ \\
\hline & WH5701_06521 & ZP_01085935 & $65353 \cdots 66231$ & 878 & hypothetical protein \\
\hline & WH5701_02369 & ZP_01084322 & $42300 \cdots 43271$ & 971 & $\operatorname{mocD}$ \\
\hline & WH5701_04005 & ZP_01083421 & $43734 \cdots 44519$ & 785 & $\mathrm{crtW}$ \\
\hline & WH5701_01215 & ZP_01084736 & $138584 \cdots 139615$ & 1031 & $\operatorname{crtR}$ \\
\hline \multirow{6}{*}{ Synechococcus sp. WH 7803} & SynWH7803_2417 & YP_001226140 & $2249293 \cdots 2250087$ & 795 & d9 \\
\hline & SynWH7803_2415 & YP_001226138 & $2247475 \cdots 2248386$ & 912 & d9 \\
\hline & SynWH7803_0589 & YP_001224312 & $594539 \cdots 595603$ & 1065 & $\mathrm{~d} 12$ \\
\hline & SynWH7803_1625 & YP_001225348 & $1496144 \cdots 1497139$ & 996 & $\mathrm{~d} 15$ \\
\hline & SynWH7803_0928 & YP_001224651 & $871421 \cdots 872167$ & 747 & crtW \\
\hline & SynWH7803_0337 & YP_001224060 & $361336 \cdots 362337$ & 1002 & $\operatorname{crtR}$ \\
\hline
\end{tabular}


Table 1: Continued.

\begin{tabular}{|c|c|c|c|c|c|}
\hline Species & Locus tag & Accession & DNA coordinates & Length & Proposed function \\
\hline \multirow{6}{*}{ Synechococcus sp. WH 7805} & WH7805_10184 & ZP_01125021 & $209067 \cdots 209999$ & 932 & $\mathrm{~d} 9$ \\
\hline & WH7805_10194 & ZP_01125023 & $210769 \cdots 211680$ & 911 & d9 \\
\hline & WH7805_06186 & ZP_01124768 & $405535 \cdots 406059$ & 524 & $\mathrm{~d} 12$ \\
\hline & WH7805_04931 & ZP_01124517 & $184338 \cdots 185516$ & 1178 & $\mathrm{~d} 12$ \\
\hline & WH7805_01197 & ZP_01123773 & $3991 \cdots 4734$ & 743 & $\operatorname{crtW}$ \\
\hline & WH7805_07481 & ZP_01123496 & $193165 \cdots 194193$ & 1028 & crtR \\
\hline \multirow{5}{*}{ Synechococcus sp. WH 8102} & SYNW2377 & NP_898466 & $2286168 \cdots 2287028$ & 860 & $\mathrm{~d} 9$ \\
\hline & SYNW0696 & NP_896789 & $679330 \cdots 680478$ & 1148 & $\mathrm{~d} 12$ \\
\hline & SYNW1696 & NP_897787 & $1631011 \cdots 1632147$ & 1136 & $\mathrm{~d} 12$ \\
\hline & SYNW1368 & NP_897461 & $1354793 \cdots 1355527$ & 734 & $\operatorname{crtW}$ \\
\hline & SYNW0291 & NP_896386 & $291323 \cdots 292354$ & 1031 & $\operatorname{crtR}$ \\
\hline \multirow{6}{*}{ Synechocystis sp. PCC 6803} & sll0541 & NP_442430 & $2822579 \cdots 2823535$ & 956 & d9 \\
\hline & $\operatorname{slr} 1350$ & NP_441489 & $1746308 \cdots 1747363$ & 1055 & $\mathrm{~d} 12$ \\
\hline & sll1441 & NP_441622 & $1895520 \cdots 1896599$ & 1079 & $\mathrm{~d} 15$ \\
\hline & sll0262 & NP_441824 & $2120067 \cdots 2121146$ & 1079 & $\mathrm{~d} 6$ \\
\hline & S1l1611 & NP_441220 & $1462136 \cdots 1463245$ & 1110 & hypothetical protein \\
\hline & sll1468 & NP_440788 & $981691 \cdots 982629$ & 938 & crtR \\
\hline \multirow{5}{*}{$\begin{array}{l}\text { Thermosynechococcus } \\
\text { elongatus strain BP-1 }\end{array}$} & tll1719 & NP_682509 & $1800682 \cdots 1801521$ & 839 & d9 \\
\hline & $\operatorname{tl} 2380$ & NP_683170 & $2490209 \cdots 2491048$ & 839 & d9 \\
\hline & $\operatorname{tlr} 1653$ & NP_682443 & $1733919 \cdots 1734767$ & 848 & d9 \\
\hline & $\operatorname{tl} 1254$ & NP_682044 & $1300388 \cdots 1301308$ & 920 & mocD \\
\hline & $\operatorname{tlr} 1900$ & NP_682690 & $1986642 \cdots 1987529$ & 887 & crtR \\
\hline \multirow{5}{*}{$\begin{array}{l}\text { Trichodesmium erythraeum } \\
\text { IMS101 }\end{array}$} & Tery_1437 & YP_721205 & $2173203 \cdots 2174015$ & 812 & d9 \\
\hline & Tery_0142 & YP_720110 & $207806 \cdots 208861$ & 1055 & $\mathrm{~d} 12$ \\
\hline & Tery_4492 & YP_723951 & $6931402 \cdots 6932475$ & 1073 & $\mathrm{~d} 15$ \\
\hline & Tery_3898 & YP_723406 & $6024293 \cdots 6025342$ & 1050 & hypothetical protein \\
\hline & Tery_2925 & YP_722564 & $4543239 \cdots 4544114$ & 875 & crtR \\
\hline \multirow{7}{*}{ Lyngbya sp. PCC 8106} & L8106_03152 & ZP_01624678 & $2253 \cdots 3071$ & 818 & d9 \\
\hline & L8106_27002 & ZP_01621185 & $94912 \cdots 95955$ & 1043 & $\mathrm{~d} 12$ \\
\hline & L8106_10697 & ZP_01624560 & $6961 \cdots 8043$ & 1082 & $\mathrm{~d} 15$ \\
\hline & L8106_14825 & ZP_01619238 & $100018 \cdots 101133$ & 1115 & $\mathrm{~d} 6$ \\
\hline & L8106_06180 & ZP_01620148 & $172993 \cdots 173604$ & 611 & hypothetical protein \\
\hline & L8106_18641 & ZP_01624278 & $13290 \cdots 14111$ & 821 & hypothetical protein \\
\hline & L8106_30215 & ZP_01622578 & $23391 \cdots 24185$ & 794 & crtR \\
\hline \multirow{8}{*}{$\begin{array}{l}\text { Nodularia spumigena } \\
\text { CCY } 9414\end{array}$} & N9414_19077 & ZP_01631817 & $16235 \cdots 17026$ & 791 & d9 \\
\hline & N9414_07494 & ZP_01632615 & $317 \cdots 1135$ & 818 & d9 \\
\hline & N9414_07499 & ZP_01632616 & $1303 \cdots 2427$ & 1124 & $\mathrm{~d} 12$ \\
\hline & N9414_07504 & ZP_01632617 & $2618 \cdots 3688$ & 1070 & $\mathrm{~d} 15$ \\
\hline & N9414_07509 & ZP_01632618 & $4087 \cdots 5178$ & 1091 & d6 \\
\hline & N9414_18293 & ZP_01629726 & $29633 \cdots 30223$ & 590 & hypothetical protein \\
\hline & N9414_07726 & ZP_01632305 & $4851 \cdots 5633$ & 782 & crtW \\
\hline & N9414_01572 & ZP_01632726 & $697 \cdots 1587$ & 890 & $\operatorname{crtR}$ \\
\hline
\end{tabular}


TABle 1: Continued.

\begin{tabular}{|c|c|c|c|c|c|}
\hline Species & Locus tag & Accession & DNA coordinates & Length & Proposed function \\
\hline \multirow{9}{*}{ Cyanothece sp. CCY0110 } & CY0110_10577 & ZP_01726409 & $185891 \cdots 186724$ & 834 & $\mathrm{~d} 9$ \\
\hline & CY0110_05582 & ZP_01729213 & $74180 \cdots 75004$ & 825 & d9 \\
\hline & CY0110_10917 & ZP_01732458 & $7951 \cdots 9000$ & 1050 & $\mathrm{~d} 12$ \\
\hline & CY0110_00445 & ZP_01728541 & $90142 \cdots 91191$ & 1050 & $\mathrm{~d} 15$ \\
\hline & CY0110_24056 & ZP_01727982 & $158769 \cdots 159887$ & 1119 & d6 \\
\hline & CY0110_13441 & ZP_01729024 & $60390 \cdots 61220$ & 831 & hypothetical protein \\
\hline & CY0110_27283 & ZP_01731934 & $15787 \cdots 16914$ & 1128 & hypothetical protein \\
\hline & CY0110_11357 & ZP_01729279 & $9512 \cdots 10513$ & 1002 & $\operatorname{mocD}$ \\
\hline & CY0110_08481 & ZP_01731007 & $25752 \cdots 26747$ & 996 & crtR \\
\hline
\end{tabular}

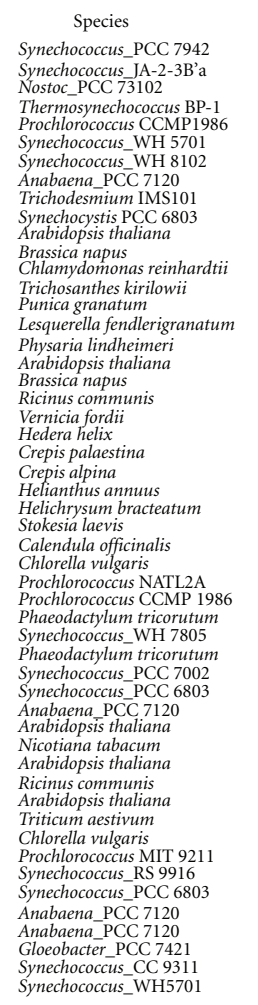

Accession number
YP_400730
YP_479096
ZP_0109371
NP_6820044
NP_893495
ZP_1086617
NP_897787
NP_485638
YP-720110
YP441489
AAA92800
AAA50157
AB007640
AAO37751
AAO37553
AAC32755
AAQ01458
NP_187819
AAF78778
AAC49010
AAN87574
AAO38031
CAA76156
CAA76158
AAO38032
AAO38037
AAR23815
AAK26632
AB075526
YP_91588
YP_893499
AAO23565
ZP_01124517
AY165023
AAB61352
NP_441622
NP_485637
BAA05514
BAC01273
AAB60302
AAA73511
BAC01274
BAA28358
AB075527
ZP_01006041
ZP-01472905
NP_440788
NP-488049
NP_487229
NP_924674
YP_731008
ZP_01083421
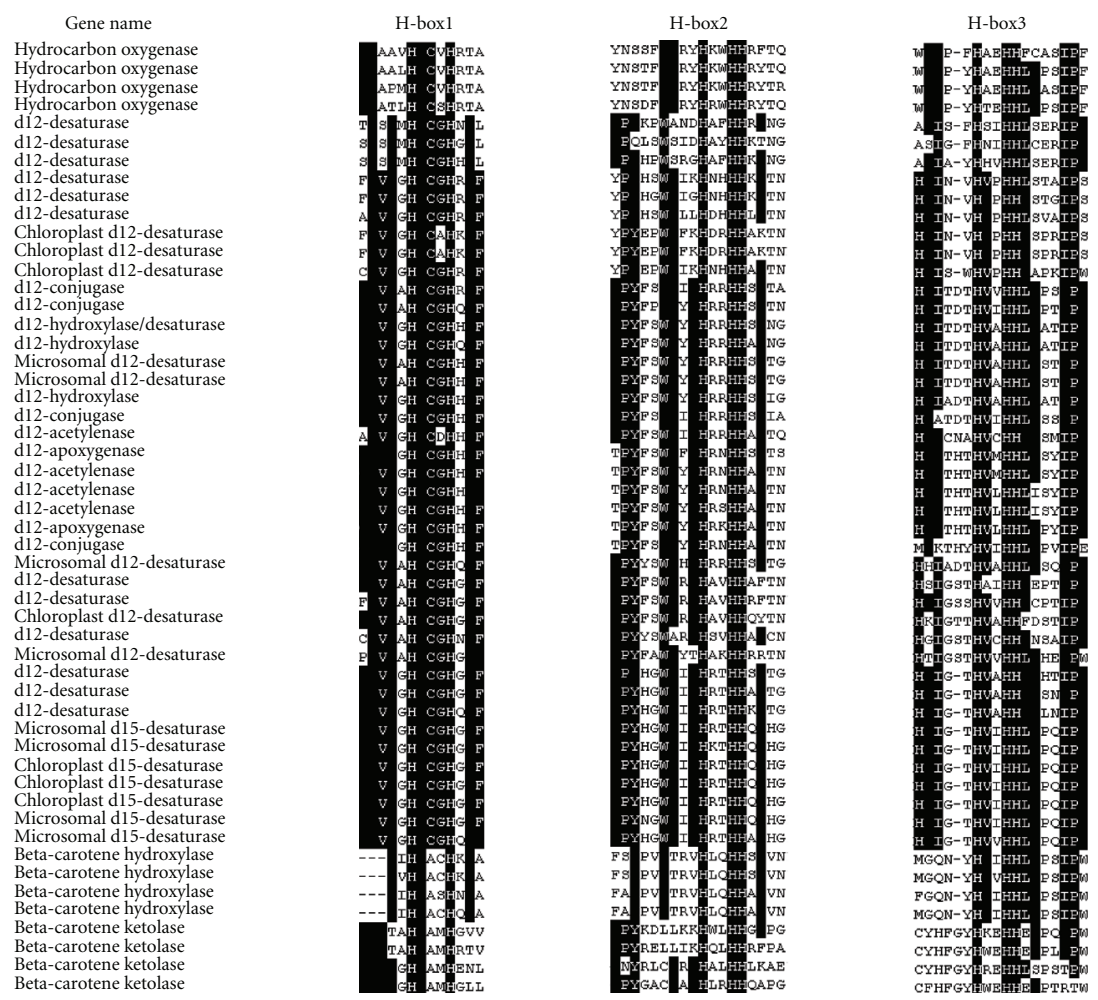

FIGURE 2: Comparison of the three conserved histidine-rich motifs of proteins from cyanobacteria, eukaryotic algae, and higher plants, including $\Delta 12$ fatty acid desaturase, $\Delta 15$ fatty acid desaturase, $\beta$-carotene ketolase, $\beta$-carotene hydroxylase, hydrocarbon oxygenase, $\Delta 12$ fatty acid epoxygenase, $\Delta 12$ fatty acid acetylenase, $\Delta 12$ fatty acid conjugase, and $\Delta 12$ fatty acid hydroxylase. The conserved amino acid residues are in black. "Microsomal" represents the microsome-type desaturases, "Chloroplast" represents the chloroplast-type desaturases.

were analyzed using neighbor-joining (NJ) and minimumevolution (ME) methods. Observation of the tree revealed that all the desaturases fell into three distinct subfamilies (Figures 12 and 13): $\Delta 9$ desaturase subfamily, $\Delta 12 / \omega 3$ desaturases subfamily, and the front-end desaturases subfamily.

As shown in Figures 12 and 13, $\Delta 9$ desaturases clustered into a single-monophyletic group, thus were analyzed separately from other types of desaturases. Six clades could be identified within the $\Delta 9$ desaturase homologs from cyanobacteria based on high-bootstrap support values and a large degree of within-clade sequence identity (Figures 3, 6, and 7). Except for the genes from Clade 6 (ZP_01620148, ZP_01085935, and AAF21447) whose second residue of the second histidine-box was not arginine, the genes from other clades all matched the standard for $\Delta 9$ desaturase, that is, $\mathrm{HR}_{3}-\mathrm{X}, \mathrm{HR}-\mathrm{X}-\mathrm{HH}$, and HN-X-HH. Thus, genes from Clade 6 are assigned as hypothetical proteins with functions unknown.

The first clade was composed by one $\Delta 9$-homologous gene from eight $\mathrm{N}_{2}$-fixing cyanobacterial species (such as 
TABLE 2: List of organisms (except the above thirty seven cyanobacteria) and protein sequences analyzed in this study. Note: micro represents Microsomal, chl represents Chloroplastic, "uncertain" means that the function of the gene is uncertain.

\begin{tabular}{|c|c|c|c|c|}
\hline Species & Accession no/locus tag & Label & Accession no/locus tag & Label \\
\hline \multirow{4}{*}{ Arabidopsis thaliana } & BAA25180 & d9 & AAB60302 & chld15 \\
\hline & Q949X0 & $\mathrm{d} 7$ & BAA05514 & $\operatorname{microd} 15$ \\
\hline & AAA92800 & chld12 & CAA11858 & $\mathrm{d} 8$ \\
\hline & NP_187819 & $\operatorname{microd} 12$ & & \\
\hline \multirow{4}{*}{ Thalassiosira pseudonana } & Tp22511 & $\mathrm{d} 9$ & AY817152 & $\mathrm{d} 5$ \\
\hline & Tp23798 & $\mathrm{d} 12$ & AY817155 & d6 \\
\hline & Tp3143 & $\mathrm{d} 12$ & AY817154 & $\mathrm{d} 8$ \\
\hline & AY817156 & $\mathrm{d} 4$ & & \\
\hline \multirow{3}{*}{ Phaeodactylum tricorutum } & AAW70158 & d9 & AY082393 & d6 \\
\hline & $\mathrm{AAO} 23565$ & chld12 & AY082392 & $\mathrm{d} 5$ \\
\hline & AY165023 & $\operatorname{microd} 12$ & Pt22459 & $\mathrm{d} 5$ \\
\hline \multirow{3}{*}{ Chlamydomonas reinhardtii } & Cr117883 & uncertain & ABL09485 & $\mathrm{d} 15$ \\
\hline & AB007640 & chld12 & AY860820 & crtW \\
\hline & EDP04777 & $\operatorname{microd} 12$ & & \\
\hline \multirow{2}{*}{ Synechococcus sp. PCC 7002} & AAB61353 & d9 & AAF21445 & $\mathrm{d} 12$ \\
\hline & AAF21447 & uncertain & AAB61352 & $\mathrm{d} 15$ \\
\hline \multirow{2}{*}{ Nostoc sp. SO-36 } & CAF18426 & $\mathrm{d} 9$ & CAF18425 & $\mathrm{d} 15$ \\
\hline & CAF18423 & d9 & CAF18424 & $\mathrm{d} 12$ \\
\hline \multirow{2}{*}{ Mortierella alpina } & CAB38177 & d9 & AAF08684 & $\mathrm{d} 12$ \\
\hline & AAF08685 & d6 & AAC39508 & $\mathrm{d} 5$ \\
\hline \multirow{2}{*}{ Cyanidioschyzon merolae } & BAA28834 & d9 & CMK291C & $\mathrm{d} 12$ \\
\hline & CMJ201C & d9 & BAC76126 & crtR \\
\hline \multirow{2}{*}{ Arthrospira platensis } & CAA05166 & d9 & Q54794 & $\mathrm{d} 12$ \\
\hline & ABN11122 & d6 & & \\
\hline \multirow{2}{*}{ Ostreococcus lucimarinus } & Ol51664 & uncertain & $\mathrm{Ol} 24150$ & $\mathrm{~d} 12$ \\
\hline & Ol18582 & $\mathrm{d} 12$ & & \\
\hline \multirow{2}{*}{ Caenorhabditis elegans } & AAF97550 & d9 & AAC15586 & d6 \\
\hline & AAC95143 & $\mathrm{d} 5$ & & \\
\hline \multirow{2}{*}{ Rattus norvegicus } & NP_114029 & d9 & BAA75496 & d6 \\
\hline & AAG35068 & $\mathrm{d} 5$ & & \\
\hline \multirow{2}{*}{ Homo sapiens } & XP_005719 & d9 & AAD20018 & d6 \\
\hline & AAF29378 & $\mathrm{d} 5$ & & \\
\hline \multirow{2}{*}{ Brassica napus } & AAA50157 & chl d12 & AAF78778 & $\operatorname{microd} 12$ \\
\hline & CAA11857 & $\mathrm{d} 8$ & & \\
\hline Chlorella vulgaris & AB075526 & $\operatorname{microd} 12$ & AB075527 & $\operatorname{microd} 15$ \\
\hline Chlamydomonas sp. W80 & AB031546 & chld12 & & \\
\hline Synechocystis sp. PCC 6714 & BAA02921 & $\mathrm{d} 12$ & & \\
\hline Mucor circinelloides & AAD55982 & $\mathrm{d} 12$ & BAB69055 & d6 \\
\hline Emericella nidulans & AAG36933 & $\mathrm{d} 12$ & & \\
\hline Glycine max & BAD89862 & $\operatorname{microd} 12$ & & \\
\hline Calendula officinalis & AAK26633 & $\operatorname{microd} 12$ & & \\
\hline Gossypium hirsutum & AAL37484 & $\operatorname{microd} 12$ & & \\
\hline Nicotiana tabacum & BAC01274 & chld15 & BAC01273 & $\operatorname{microd} 15$ \\
\hline Brassica juncea & CAB85467 & chld15 & & \\
\hline Picea abies & CAC18722 & chld 15 & & \\
\hline Ricinus communis & AAA73511 & chld15 & AAC49010 & 12-hydroxylase \\
\hline Triticum aestivum & BAA28358 & $\operatorname{microd} 15$ & & \\
\hline Oryza sativa & BAA11397 & $\operatorname{microd} 15$ & & \\
\hline Vernicia fordii & AAN87573 & $\operatorname{microd} 12$ & AAN87574 & 12-conjugase \\
\hline Punica granatum & CAD24671 & $\operatorname{microd} 12$ & AAO37753 & 12-conjugase \\
\hline Lesquerella fendleri & AAC32755 & 12-hydroxylase/desaturase & & \\
\hline
\end{tabular}


Table 2: Continued.

\begin{tabular}{|c|c|c|c|c|}
\hline Species & Accession no/locus tag & Label & Accession no/locus tag & Label \\
\hline Physaria lindheimeri & $\mathrm{ABQ} 01458$ & 12-hydroxylase & & \\
\hline Crepis palaestina & CAA76156 & 12-epoxygenase & & \\
\hline Stokesia laevis & AAR23815 & 12-epoxygenase & & \\
\hline Daucus carota & AAO38033 & 12-acetylenase & & \\
\hline Foeniculum vulgare & AAO38034 & 12-acetylenase & & \\
\hline Hedera helix & AAO38031 & 12-acetylenase & & \\
\hline Helianthus annuus & AAO38032 & 12-acetylenase & CAA60621 & $\mathrm{d} 8$ \\
\hline Helichrysum bracteatum & AAO38037 & 12-acetylenase & & \\
\hline Rudbeckia hirta & AAO38035 & 12-acetylenase & & \\
\hline Crepis alpina & CAA76158 & 12-acetylenase & & \\
\hline Calendula officinalis & AAK26632 & 12-conjugase & & \\
\hline Trichosanthes kirilowii & AAO37751 & 12-conjugase & & \\
\hline Acheta domesticus & AAK25797 & d9 & & \\
\hline Cyprinus carpio & CAB57858 & d9 & & \\
\hline Drosophila simulans & CAB52475 & d9 & & \\
\hline Gallus gallus & CAA42997 & d9 & & \\
\hline Helicoverpa zea & AAF81790 & d9 & & \\
\hline Rosa hybrid cultivar & BAA23136 & d9 & & \\
\hline Saccharomyces cerevisiae & AAA34826 & d9 & & \\
\hline Limnanthes douglasii & AAG28599 & d9 & & \\
\hline Prochlorothrix hollandica & AAG16761 & d9 & & \\
\hline Lyngbya majuscula & AAS98775 & d9 & & \\
\hline Synechococcus vulcanus & AAD00699 & d9 & & \\
\hline Thraustochytrium sp. ATCC21685 & AAM09688 & $\mathrm{d} 4$ & AAM09687 & d5 \\
\hline Euglena gracilis & AAQ19605 & $\mathrm{d} 4$ & AF139720 & $\mathrm{d} 8$ \\
\hline Pavlova lutheri & AY332747 & $\mathrm{d} 4$ & & \\
\hline Isochrysis galbana strain CCMP1323 & AY630574 & $\mathrm{d} 4$ & & \\
\hline Marchantia polymorpha & AAT85663 & d5 & AAT85661 & d6 \\
\hline Nitzschia closterium f. minutissima & AY603475 & d5 & & \\
\hline Dictyostelium discoideum & BAA37090 & d5 & & \\
\hline Bacillus subtilis & AAC38355 & d5 & & \\
\hline Danio rerio & Q9DEX7 & $\mathrm{d} 5 / \mathrm{d} 6$ & & \\
\hline Borago officinalis & AAD01410 & d6 & AAG43277 & $\mathrm{d} 8$ \\
\hline Oncorhynchus mykiss & AAK26745 & d6 & & \\
\hline Mus musculus & NP_062673 & d6 & & \\
\hline Glossomastix chrysoplasta & AAU11444 & d6 & & \\
\hline Ostreococcus tauri & AY746357 & d6 & & \\
\hline Physcomitrella patens & CAA11033 & d6 & & \\
\hline Echium pitardii & AAL23581 & d6 & & \\
\hline Chlorella zofingiensis & AY772713 & $\operatorname{crtW}$ & & \\
\hline Cyanidium caldarium & AAB82698 & $\operatorname{crtR}$ & & \\
\hline Haematococcus pluvialis & CAA60478 & crtW & & \\
\hline Myxococcus xanthus DK 1622 & YP_634431 & uncertain & & \\
\hline Stigmatella aurantiaca DW4/3-1 & ZP_01463016 & uncertain & & \\
\hline Bradyrhizobium japonicum USDA 110 & NP_771234 & uncertain & & \\
\hline
\end{tabular}

Nostoc sp. strain SO-36 and Anabaena sp. PCC 7120), Thermosynechococcus elongatus BP-1, Synechococcus vulcanus, and two genes from Gloeobacter violaceus. The amino acid identity of these genes ranged from $50 \%$ to $98 \%$ among various cyanobacterial species. It has been proven by previous research that the $\Delta 9$ desaturase gene from Nostoc sp. strain SO-36 in this clade introduced double bonds into fatty acids that are bound to the $s n-2$ position of the glycerol moiety of membrane glycerolipids [19]. Moreover, the three histidineboxes of the gene from Nostoc sp. SO-36 were consistent with 
TABle 3: Conserved motifs of membrane desaturases in cyanobacteria. Note: X represents an unspecified amino acid. $\Delta 9-1$ : clade 1 of $\Delta 9$ homologous genes, $\Delta 9-2$ : clade 2 of $\Delta 9$ homologous genes, $\Delta 9-3$ : clade 3 of $\Delta 9$ homologous genes, $\Delta 9-4$ : clade 4 of $\Delta 9$ homologous genes, $\Delta 9$-5: clade 5 of $\Delta 9$ homologous genes, $\Delta 12 \mathrm{a}$ : clade 3 of $\Delta 12$ homologous genes, $\Delta 12 \mathrm{~b}$ : clade 1 of $\Delta 12$ homologous genes, $\Delta 12 \mathrm{c}:$ clade 4 of $\Delta 12$ homologous genes, $\Delta 15: \Delta 15$ desaturase, $\Delta 6: \Delta 6$ desaturase.

\begin{tabular}{|c|c|c|c|}
\hline Name & H-boxl & H-box2 & H-box3 \\
\hline$\beta$-carotene ketolase & TGLFIX $_{2}$ HDXMH & $\mathrm{K}(\mathrm{N}) \mathrm{HX}_{2} \mathrm{HH}$ & CY(F)H(N)FGYHXEHH \\
\hline$\beta$-carotene hydroxylase & GTVIHDAS(C) $\mathrm{HX}_{2} \mathrm{AH}$ & RVHL(M)Q(E)HHXHVN & GQNYHLI(V)HHLWPSI(V)PW \\
\hline hydrocarbon oxygenase & HECXHRTAFA & FY(F)RRYHXWHHRXT & MWNMPF(Y)HXEHHL(F) \\
\hline$\Delta 9-1$ & GICLGYHRLLXHKSF & $\mathrm{WX}_{3} \mathrm{HRXHHAX}_{3} \mathrm{D}$ & YGEGWHNNHHX ${ }_{2} \mathrm{PX}_{5} \mathrm{GX}_{2}$ WWE \\
\hline$\Delta 9-2$ & GXTLGXHRX $_{3} \mathrm{HRSF}$ & WXGXHRXHHX ${ }_{2} \mathrm{SD}$ & GEGWHNNHHX $_{4}$ SARHGXXWWE \\
\hline$\Delta 9-3$ & TVLGVTLGLHRLXAHRS & $\mathrm{WX}_{2} \mathrm{LHRHHHX}_{2} \mathrm{SDQ}$ & WVAXLSFGEGWHNNHHAXPXSARHGL \\
\hline$\Delta 9-4$ & CLGVTXGYHRLLXHRX $_{2}$ & WXGLHRHHHXFSDT & WVAALTFGEGWHNNHHAXPXSA \\
\hline$\Delta 9-5$ & $\mathrm{GX}_{4} \mathrm{GXHRXFXHX}_{2} \mathrm{~F}$ & $\mathrm{WX}_{3} \mathrm{HRXHHX}_{3} \mathrm{D}$ & GESWHNNHHXFX $_{3} \mathrm{AX}_{2} \mathrm{G}$ \\
\hline$\Delta 12 \mathrm{a}$ & FVXGHDCGHRSF & $\mathrm{WRX}_{2} \mathrm{HX}_{2} \mathrm{HHX}_{2} \mathrm{TN}$ & $\mathrm{HXPHHX}_{4} \mathrm{IPXYNLR}$ \\
\hline$\Delta 12 \mathrm{~b}$ & WVXAHECGHXAFH & $\mathrm{WX}_{2} \mathrm{SHX}_{2} \mathrm{HHX}_{3} \mathrm{~N}$ & $\mathrm{HX}_{2} \mathrm{HHX}_{4} \mathrm{PHYXA}$ \\
\hline$\Delta 12 \mathrm{c}$ & FSLMHDCGHXSLF & $\mathrm{WSX}_{2} \mathrm{HAXHHX}_{2} \mathrm{NG}$ & HX $_{2}$ HHLXERIPNYXL \\
\hline$\Delta 15$ & FWXLFVVGHDCGHXSFS & HGWRISHRTHHXNTGN & IHHXIGTHVAHHIF \\
\hline$\Delta 6$ & $\mathrm{HDX}_{2} \mathrm{HX}_{3} \mathrm{~S}$ & $\mathrm{WX}_{3} \mathrm{HX}_{2} \mathrm{LHHXYTNI}$ & GGLNXQ(H)X ${ }_{2}$ HHLFPXICH \\
\hline
\end{tabular}

those of genes in Clade 1. Therefore, the genes of Clade 1 are presumed to act on fatty acids esterified to the $s n-2$ position of glycerolipids.

In Clade 2, one $\Delta$ 9-homologous gene from Prochlorothrix hollandica, Synechococcus sp. PCC 7942, and Synechococcus sp. PCC 6301 clustered together with two genes from Thermosynechococcus elongatus, apart from the subgroup comprised of genes from nine $\mathrm{N}_{2}$-fixing cyanobacterial species (such as Anabaena variabilis and Trichodesmium erythraeum), Synechocystis sp. PCC 6803, Synechococcus sp. PCC 7002, and Arthrospira platensis. It has been demonstrated that Thermosynechococcus elongatus has three $\Delta 9$-homologous genes that consist of one c-type and two unspecified types. By contrast, Synechococcus sp. PCC 7942, Synechococcus sp. PCC 6301, and Prochlorothrix hollandica have only one $\Delta 9$ homologous gene, which is nonspecific with respect to $s n$ positions, acting on fatty acids at both the $s n-1$ and $s n-2$ positions [19]. $\Delta 9$ homologs from another subgroup showed high similarity with amino acid identity from 53\% to $98 \%$ among various cyanobacterial species. They are strongly homologous to the genes of Synechocystis sp. PCC 6803 (NP_442430), Synechococcus sp. PCC 7002 (AAB61353), and Arthrospira platensis (CAA05166) that encode $\Delta 9$ desaturases acting on C18 fatty acids at the $s n-1$ position. Moreover, the three histidine-boxes of these $\Delta 9$-homologous genes ( HRX $_{3}$ HRSF, WXGXHRXHH, GEGWHNNHH) accorded with those inferred by Chintalapati et al. (2006) [19].

The $\Delta 9$-homologous genes from two unicellular marine cyanobacteria Synechococcus and Prochlorococcus constituted the third and fourth clades. Amino acid identity of genes from these two clades ranged from $54 \%$ to $98 \%$ and $65 \%$ to $99 \%$, respectively. In addition, the two groups are closely related to Clade 2. Therefore, it is possible that these genes are homologous to the gene that encodes a $\Delta 9$ desaturase acting on C18 fatty acids at the $s n-1$ position or $s n-1$ and $s n$ 2 positions of glycerolipids. In these two clades, 11 strains (nine Synechococcus and two low light-adapted Prochlorococcus strains) contained two $\Delta 9$-homologous genes, which clustered separately into two subgroups. It is possible that there are two paralogous genes of a common ancestor in some evolutionary lineages, such as Synechococcus sp. CC9605; however, one of them has been lost. Alternatively, acquirement of one gene from other organisms could have occurred in the evolutionary lineage, in which horizontal gene transfer (HGT) might have taken place.

Four genes of Gloeobacter violaceus PCC 7421 as well as JamB gene of Lyngbya majuscula integrated the fifth clade. $\mathrm{JamB}$ is a gene of jamaicamide biosynthetic gene cluster, and similar to a large family of membrane-associated desaturases that utilize a diiron active site to execute $\Delta 5$ - or $\Delta 9$-fatty acid desaturation [20]. These genes fell into the group of proteobacterial stearoyl-CoA desaturases, far away from the other desaturase genes of cyanobacteria as analyzed by BLASTP program of NCBI (data not shown). It is probable that horizontal gene transfer (HGT) from other organisms like proteobacteria might have occurred.

Phylogenetic analyses from Figures 12 and 13 showed that $\Delta 9$ desaturases from cyanobacteria were grouped to those from green algae and higher plants, apart from red algae, diatoms, fungi, and animals. Among cyanobacterial $\Delta 9$ desaturases, the desaturase genes acting on fatty acids esterified to the $s n-1$ or $s n-1$ and $s n-2$ positions of glycerolipids (b-type or a-type) were placed in a basal position, while desaturase genes acting on fatty acids esterified to the $s n-2$ position of glycerolipids (c-type) were in the exoteric position, which indicates that a-type or b-type $\Delta 9$ desaturases may be ancestral to c-type desaturase. 
Nodularia_CCY9414_clade 1 Synechococcus_JA-2-3B'a_clade 1 Thermosynechococcus BP-1 clade 1 Nostoc_36_clade 2

Arthrospira platensis clade 2 Synechocystis_PCC6803 clade 2 Synechococcus_PCC7942 clade 2 Prochlorothrix_clade 2

Thermosynechococcus BP-1 clade 2 Prochlorococcus_MIT9313 clade 4 Synechococcus_RS9916_clade 4 Prochlorococcus 1986 clade 4 Prochlorococcus_MIT9303 clade 3 Synechococcus_CC9605 clade 3 Synechococcus_WH7805_clade 3 Synechococcus_WH5701 clade 6 Synechococcus_PCC7002 clade 6 Gloeobacter_PCC7421_clade 5 Gloeobacter PCC7421 clade 5 Gloeobacter_PCC7421_clade 5

Nodularia_CCY9414_clade 1 Synechococcus_JA-2-3B'a_clade 1 ThermosynechococcusBP-1 clade 1 Nostoc_36_clade 2

Arthrospira_platensis_clade 2 Synechocystis_PCC6803_clade 2 Synechococcus_PCC7942_clade 2

Thermosynechococcus BP-1 clade 2 Prochlorococcus_MIT9313_clade Synechococcus_RS9916_clade Prochlorococcus_1986_clade 4 Prochlorococcus_MTI9303_clade 3 Synechococcus_CC9605_clade 3 Synechococcus_WH7805_clade 3 Symechoc_. Synechococtus_PCC7002_clade 6 Gloobucter_PC7421_c Gloeob Gloeobacter_PCC7421_clade 5

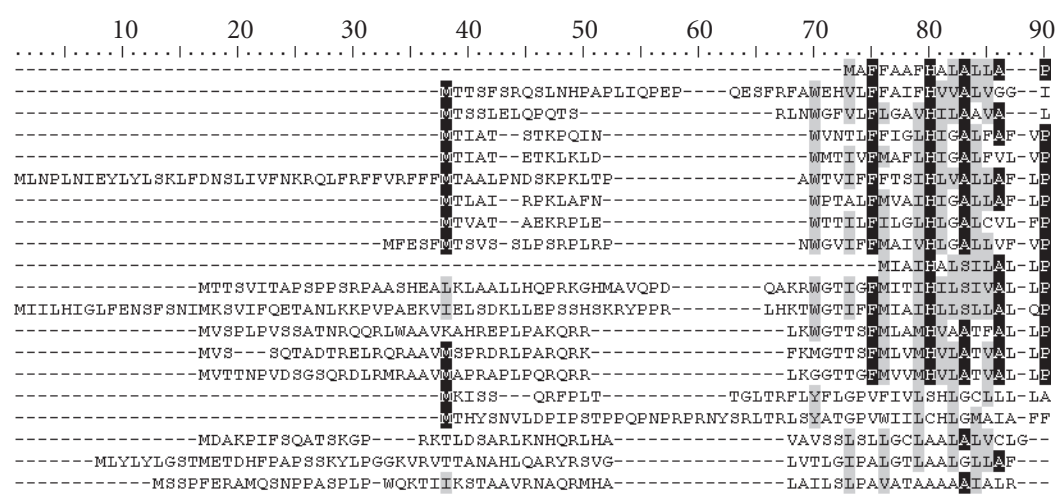

(a)

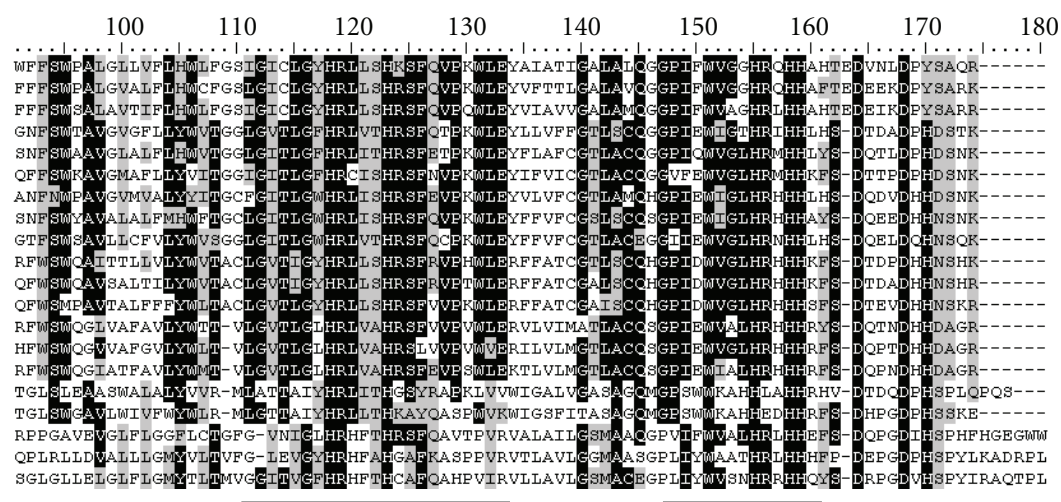

(b)

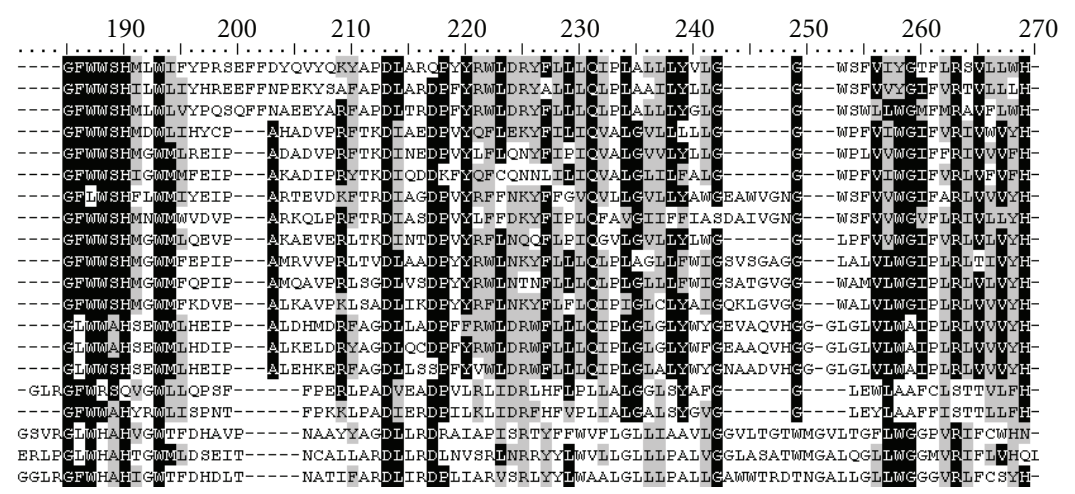

(c)

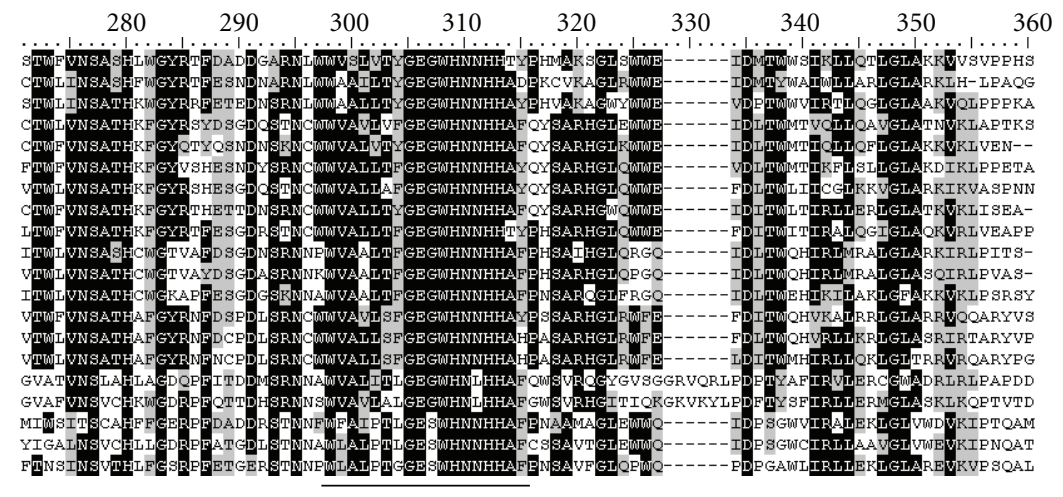

(d) Nodularia_CCY9414_clade 1
Synechococcus_JA-2-3B'a_clade 1
ThermosynechococcusBP-1 clade 1
Nostoc_36_clade 2
Arthrospira_platensis_clade 2
Synechocystis_PCC6803_clade 2
Synechococcus_PCC7942_clade 2
Prochlorothri__clade 2
ThermosynechococcusBP-1 clade 2
Prochlorococcus_MIT9313_clade 4
Synechococcus_RS9916_clade 4
Prochlorococcus_1986_clade 4
Prochlorococcus_MIT9303_clade 3
Synechococcus_CC9605_clade 3
Synechococcus_WH7805_clade 3
Synechooccus_WH5701_clade 6
Synechococcus_PCC7002_clade 6
Gloeobacter_PCC7421_clade 5
Gloeobacter_PCC7421_clade 5
Gloeobacter_PCC7421_clade 5

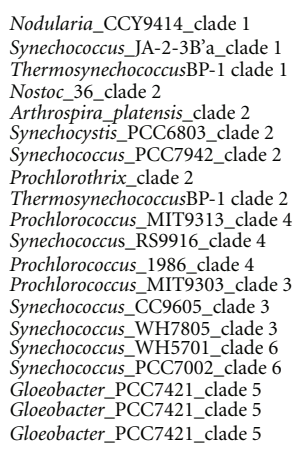

Nodularia_CCY9414_clade Thechococas_JA-2-3B' a Nostoc_36_clade 2

Synrospir_platensis_clade 2 Synechococcus_PCC7942_clade 2 ThermosynechococusBP-1

Prochlorocus

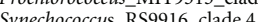
Prochlorococcus_1986_clade 4

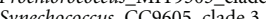

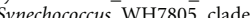
Synechococcus_WH5701_clade 6 Shechoci Gloeobacter_PCC7421_clade 5

FIGURE 3: Alignment of the complete deduced amino acid sequences of $\Delta 9$-homologous genes. Amino acid residues that are conserved are highlighted in black boxes. The conserved His clusters and their associated conserved domains are underlined. The limits of the domains are indicated by the residue positions, on top of the sequence. The sequences are denoted by their strain names and the clades they belong to. 


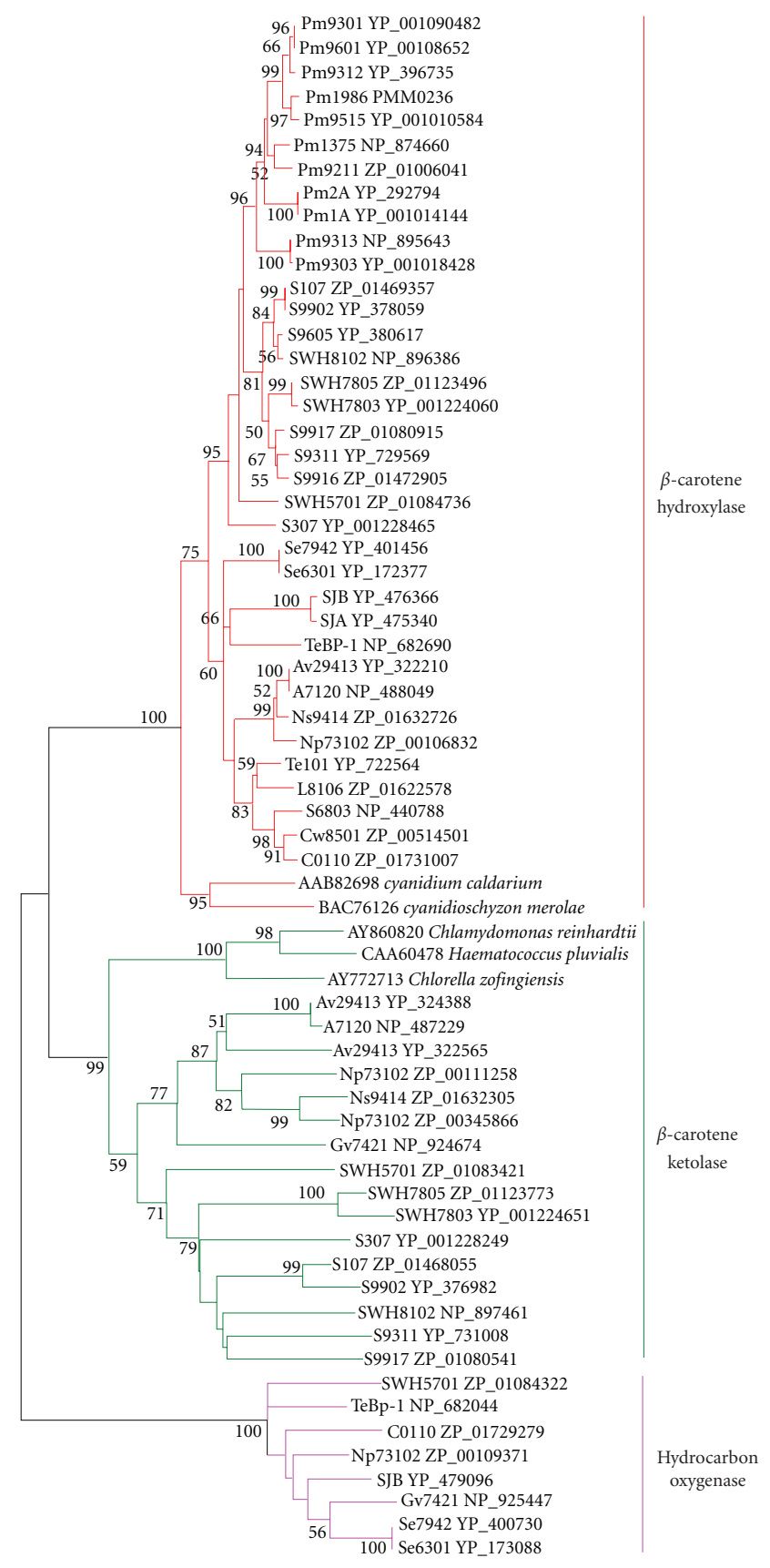

FIGURE 4: Neighbor-joining tree of $\beta$-carotene ketolase, $\beta$-carotene hydroxylase, and hydrocarbon oxygenase homologs of cyanobacteria and eukaryotic algae. About 220 positions spanning the three histidine-boxes were employed. Colored branches indicate different groups of proteins. Red: $\beta$-carotene hydroxylase, green: $\beta$-carotene ketolase, magenta: hydrocarbon oxygenase. Sequences from 37 sequenced cyanobacterial genomes are shown by their acronyms and accession numbers (locus tags). Other sequences are shown by their accession numbers, labels, and strain names. Desaturase genes that have been functionally characterized are indicated on the tree by their labels. Bootstrap values from neighbor-joining analyses are listed to the left of each node, with values more than 50 are shown.

\subsection{Discovery of Candidate Genes for $\Delta 12 / \omega 3$ Desaturases}

Observation on the phylogenetic tree of different membrane desaturases showed that $\Delta 12$ desaturases and $\Delta 15$ desaturases fell into the same clade (Figures 12 and 13), thus were analyzed together. As could be seen in Figures 8 and 9, the $\Delta 12 / \omega 3$ desaturase homologs from cyanobacteria were classified into five different clades.

It was surprising that the first clade was constituted by the $\Delta 12$ homologs of marine cyanobacteria Synechococcus, Prochlorococcus, and the microsomal $\Delta 12$ desaturases of eukaryotic algae. Moreover, three histidine-boxes of the genes from cyanobacteria were represented as AHECGH, 


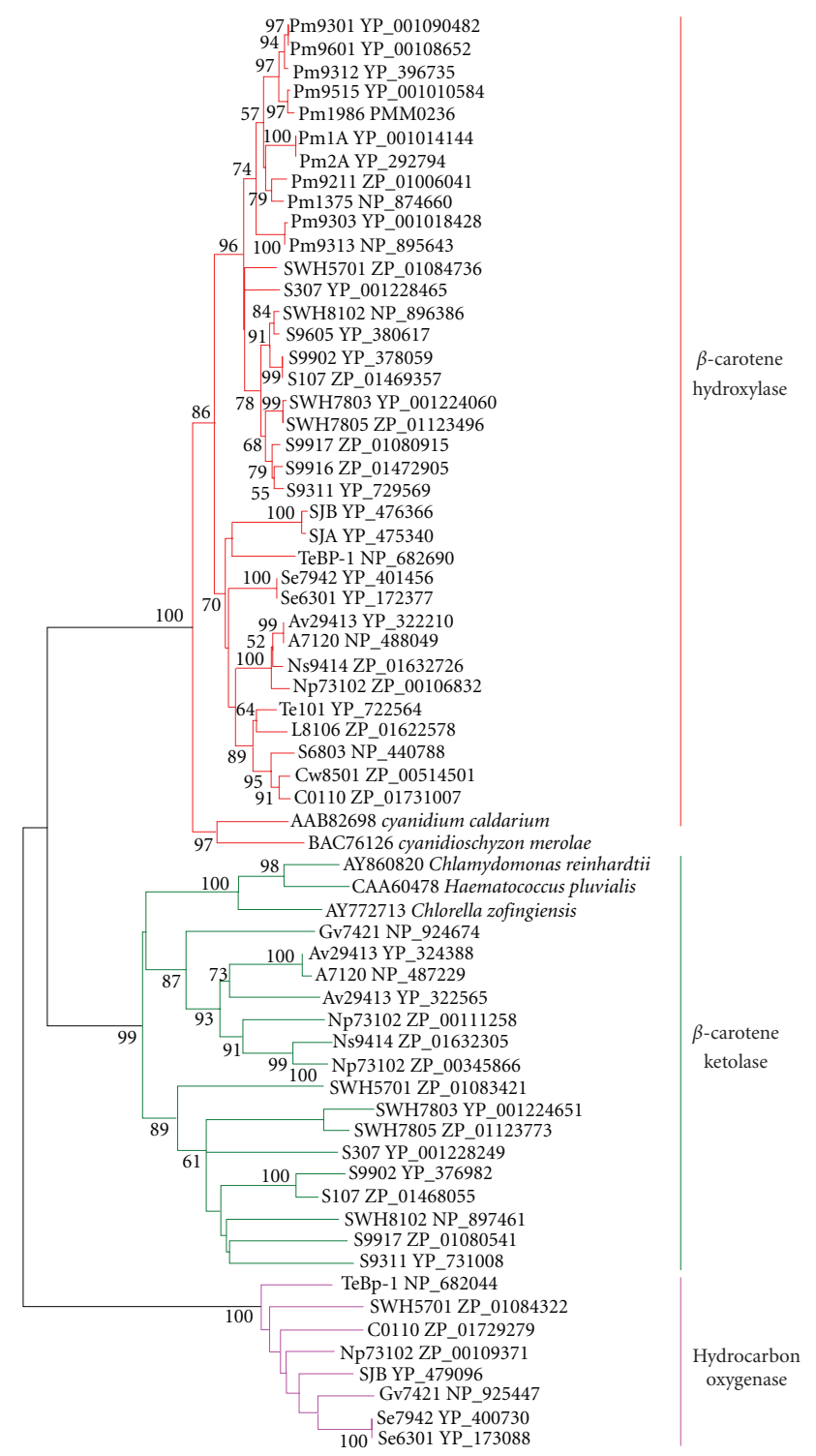

FIGURE 5: Minimum-evolution tree of $\beta$-carotene ketolase, $\beta$ carotene hydroxylase, and hydrocarbon oxygenase homologs of cyanobacteria and eukaryotic algae. About 220 positions spanning the three histidine-boxes were employed. Colored branches indicate different groups of proteins. Red: $\beta$-carotene hydroxylase, green: $\beta$-carotene ketolase, magenta: hydrocarbon oxygenase. Sequences from 37 sequenced cyanobacterial genomes are shown by their acronyms and accession numbers (locus tags). Other sequences are shown by their accession numbers, labels, and strain names. Desaturase genes that have been functionally characterized are indicated on the tree by their labels. Bootstrap values from minimum-evolution analyses are listed to the left of each node, with values more than 50 are shown.

$\mathrm{WX}_{2} \mathrm{SHX}_{2} \mathrm{HHX}_{3} \mathrm{~N}$, and $\mathrm{HX}_{2} \mathrm{HH}$ (Figure 2 and Table 3), which were similar to those of microsome-type desaturases. Two partial amino acid sequences homologous to microsome-type $\Delta 12$ desaturases were revealed in Prochlorococcus marinus MIT 9211 (ZP_01005647 and ZP_01005648). One encoded an $\mathrm{N}$-terminus region and the other encoded a
C-terminus region. They may represent a single gene inferred from their close chromosome location of the graft genome, thus were designated as a unique gene with the accession number ZP_01005647.

The microsomal $\Delta 12$ desaturases are members of a large class of membrane-bound enzymes that contain a tripartite histidine sequence motif and two putative membrane-spanning domains. This group of membranebound enzymes includes desaturases, hydroxylases, epoxygenases, acetylenases, methyl oxidases and ketolases found in animals, fungi, plants, and bacteria [21-23]. The diverse reactions that these enzymes catalyze probably use a common reactive center [24]. Histidine-rich motifs are thought to form a part of the diiron center, where oxygen activation and substrate oxidation occur [25].

To further clarify the role of genes in Clade 1, anotherphylogenetic tree was constructed by neighborjoining (NJ) and minimum-evolution (ME) methods (Figures 10 and 11). It could be seen evidently from Figures 10 and 11 that the microsomal $\Delta 12$ desaturases from higher plants and some eukaryotic algae (such as green algae, chlorella, and chlamydomonas) fell into one group with $\Delta 12$ fatty acid hydroxylase, epoxygenase, acetylenase, and conjugase, while the genes of marine cyanobacteria clustered only with diatom plastidial and microsomal $\Delta 12$ desaturases [26]. Therefore, the microsomal $\Delta 12$ desaturases of some eukaryotic algae (such as diatom) might originate from cyanobacterial orthologs in Clade 1, and possibly horizontal gene transfer might have occurred from eukaryotic algae to Synechococcus and Prochlorococcus strains.

The $\omega 3$-homologous genes of cyanobacteria and eukaryotic algae constituted the second clade. Moreover, three histidine-boxes of the genes from cyanobacteria (FVVGHDCGHXSFS, HGWRISHRTHHXNTGN, and IHHXIGTHVAHHIF) established the standard for prokaryotic $\Delta 15$ desaturase (Figure 2 and Table 3). The third clade was integrated by the $\Delta 12$ homologs of cyanobacteria and the chloroplastic $\Delta 12$ desaturases of eukaryotic algae. Moreover, three histidine-boxes of these genes were consistent with those of plastidial $\Delta 12$ desaturase that were represented as $\mathrm{HDCGH}, \mathrm{HX}_{2} \mathrm{HH}$, and HXPHH.

The homologous genes from Clade 4 also had three histidine-motifs (FSLMHDCGHXSLF, $\mathrm{WSX}_{2} \mathrm{HAXHHX}_{2}$ NG, and $\mathrm{HX}_{2}$ HHLXERIPNYXL) (Figure 2 and Table 3 ) that were similar to those of the $\Delta 12$ desaturase. As shown in Figures 12 and 13, the genes of this clade clustered with Bacillus subtilis $\Delta 5$ desaturase. Aguilar et al. (1998) demonstrated that Bacillus subtilis possessed a single desaturase. Expression of the gene in Escherichia coli resulted in desaturation of palmitic acid moieties of the membrane phospholipids to give the novel mono-UFA cis-5-hexadecenoic acid, indicating that the gene product was a $\Delta 5$ acyl-lipid desaturase [27]. However, it is well known from freshwater cyanobacteria that only four distinct desaturases, $\Delta 9, \Delta 12, \Delta 15$, and $\Delta 6$, exist in cyanobacterial cells. Therefore, the relatively close phylogenetic relationship between genes of Clade 4 and $\Delta 5$ desaturase gene of Bacillus subtilis may be due to horizontal 


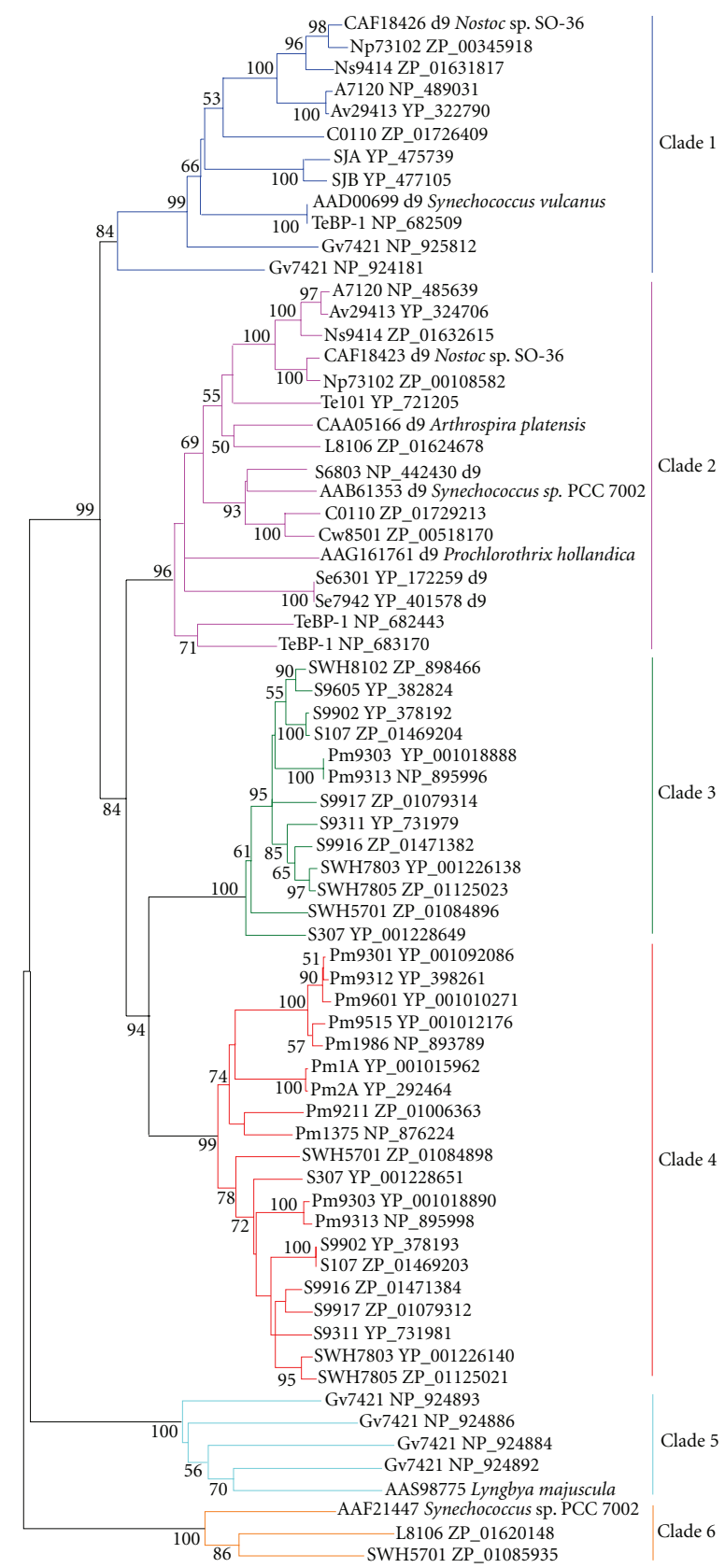

FIGURE 6: Neighbor-joining tree of $\Delta 9$-homologous genes of cyanobacteria and eukaryotic algae. About 250 positions spanning the three histidine-boxes were employed. Colored branches indicate different groups of proteins. Dark blue: Clade 1, magenta: Clade 2, green: Clade 3, red: Clade 4, light blue: Clade 5, orange: Clade 6. Sequences from 37 sequenced cyanobacterial genomes are shown by their acronyms and accession numbers (locus tags). Other sequences are shown by their accession numbers, labels, and strain names. Desaturase genes that have been functionally characterized are indicated on the tree by their labels. Bootstrap values from neighbor-joining analyses are listed to the left of each node, with values more than 50 are shown.

gene transfer and the function of these genes would require further work to fully characterize.

Three genes from Nostoc punctiforme ATCC 29133, two genes from Cyanothece sp. CCY0110, and one gene from Synechocystis sp. PCC 6803, Crocosphaera watsonii WH 8501, Lyngbya sp. PCC 8106 constituted the fifth clade. It has been proven by experiments that there is only one $\Delta 12$ desaturase in Synechocystis sp. PCC 6803 [13]. Additionally, the three histidine-motifs of these genes were $\mathrm{HXXXH}$, $\mathrm{HXXXHH,} \mathrm{HXXHH,} \mathrm{among} \mathrm{which} \mathrm{the} \mathrm{amounts} \mathrm{of} \mathrm{residues}$ between histidines from the second histidine-box were three, while that of known cyanobacterial $\Delta 12$ desaturase were two 


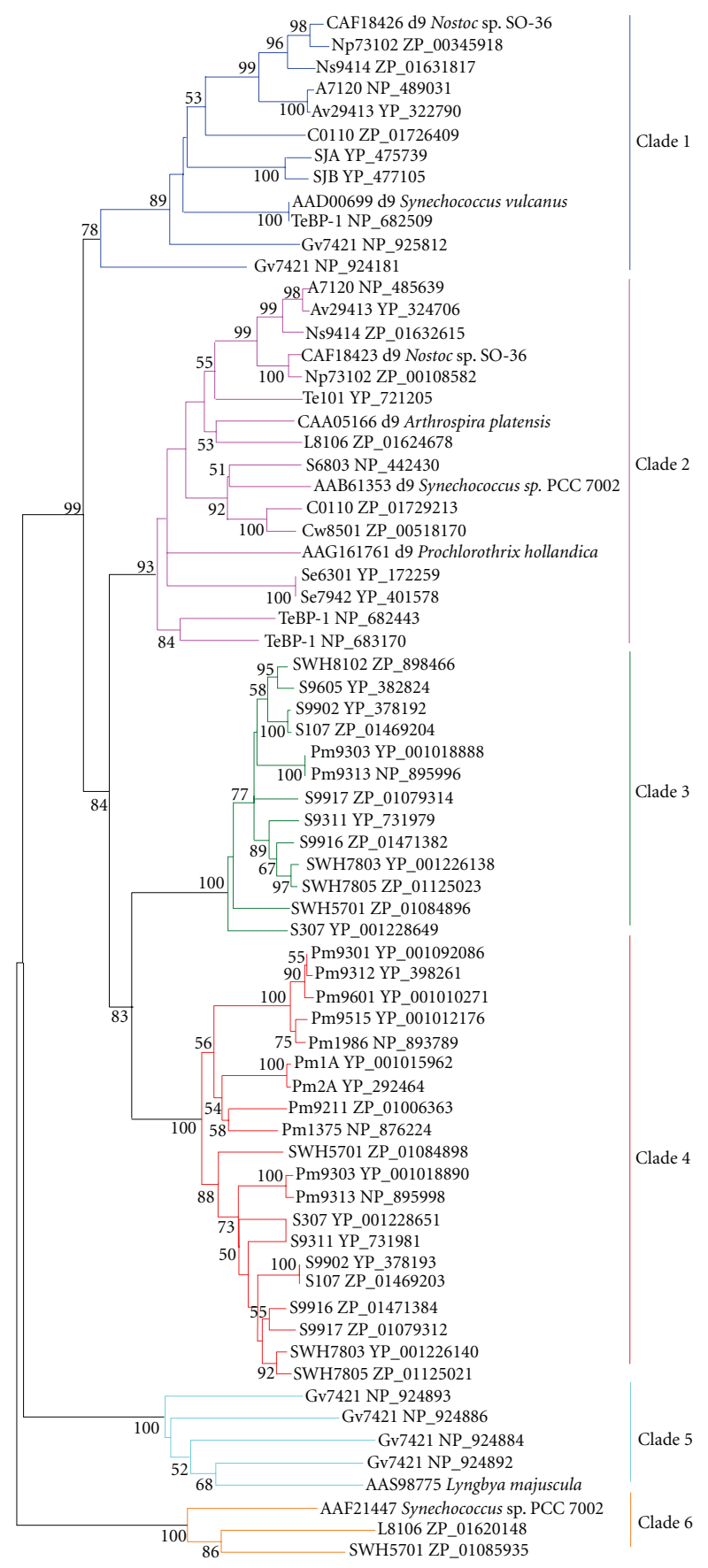

Figure 7: Minimum-evolution tree of $\Delta 9$-homologous genes of cyanobacteria and eukaryotic algae. About 250 positions spanning the three histidine-boxes were employed. Colored branches indicate different groups of proteins. Dark blue: Clade 1, magenta: Clade 2, green: Clade 3, red: Clade 4, light blue: Clade 5, orange: Clade 6. Sequences from 37 sequenced cyanobacterial genomes are shown by their acronyms and accession numbers (locus tags). Other sequences are shown by their accession numbers, labels, and strain names. Desaturase genes that have been functionally characterized are indicated on the tree by their labels. Bootstrap values from minimum-evolution analyses are listed to the left of each node, with values more than 50 are shown.

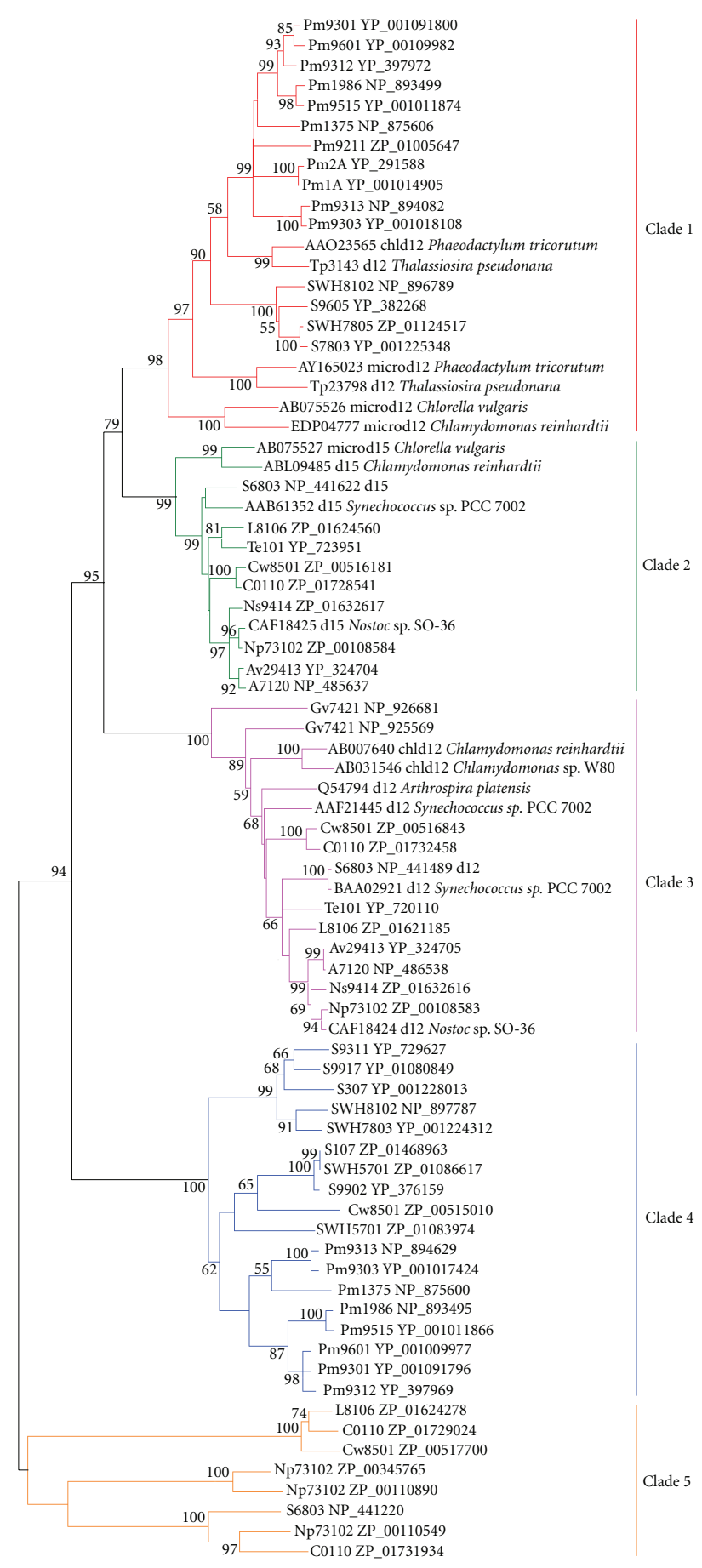

FIGURE 8: Neighbor-joining tree of $\Delta 12$ and $\Delta 15$ homologous genes of cyanobacteria and eukaryotic algae. About 300 positions spanning the three histidine-boxes were employed. Colored branches indicate different groups of proteins. Red: Clade 1, green: Clade 2, magenta: Clade 3, blue: Clade 4, orange: Clade 5. Sequences from 37 sequenced cyanobacterial genomes are shown by their acronyms and accession numbers (locus tags). Other sequences are shown by their accession numbers, labels, and strain names. Desaturase genes that have been functionally characterized are indicated on the tree by their labels. Bootstrap values from neighbor-joining analyses are listed to the left of each node, with values more than 50 are shown. 


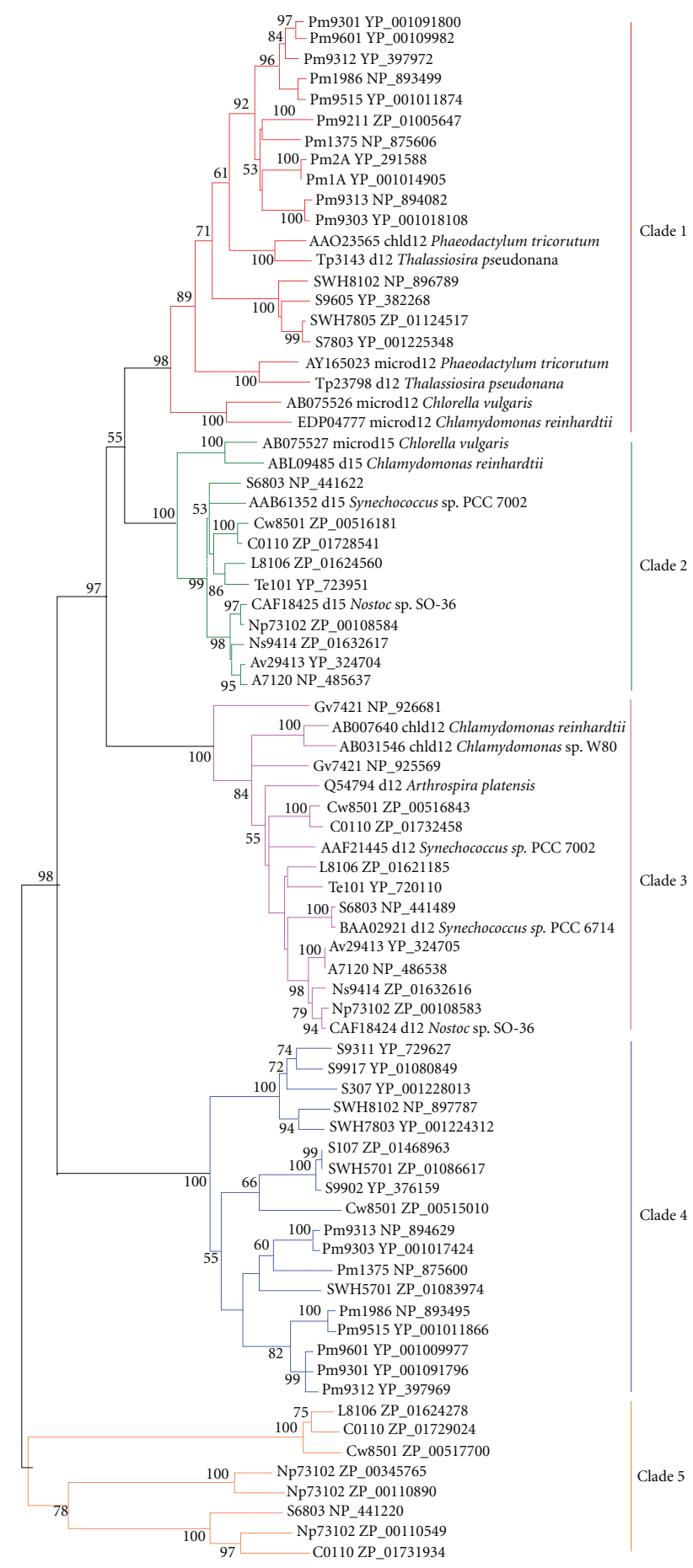

Figure 9: Minimum-evolution tree of $\Delta 12$ and $\Delta 15$ homologous genes of cyanobacteria and eukaryotic algae. About 300 positions spanning the three histidine-boxes were employed. Colored branches indicate different groups of proteins. Red: Clade 1, green: Clade 2, magenta: Clade 3, blue: Clade 4, orange: Clade 5. Sequences from 37 sequenced cyanobacterial genomes are shown by their acronyms and accession numbers (locus tags). Other sequences are shown by their accession numbers, labels, and strain names. Desaturase genes that have been functionally characterized are indicated on the tree by their labels. Bootstrap values from minimum-evolution analyses are listed to the left of each node, with values more than 50 are shown.

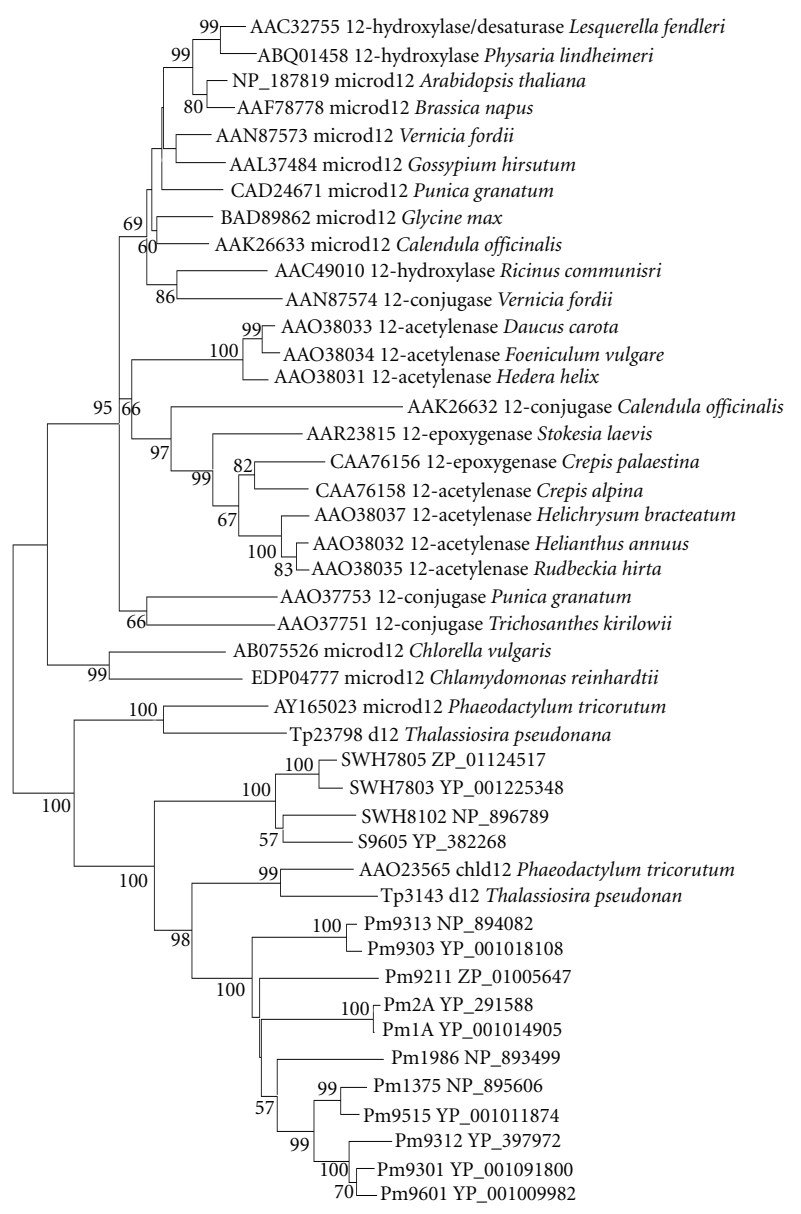

FIGURE 10: Neighbor-joining tree of $\Delta 12$ homologous genes of cyanobacteria, eukaryotic algae, and higher plants. About 300 positions spanning the three histidine-boxes were employed. Sequences from 37 sequenced cyanobacterial genomes are shown by their acronyms and accession numbers (locus tags). Other sequences are shown by their accession numbers, labels, and strain names. Desaturase genes that have been functionally characterized are indicated on the tree by their labels. Bootstrap values from neighbor-joining analyses are listed to the left of each node, with values more than 50 are shown.

(HXXXH, HXXHH, HXXHH). Therefore, in our analysis they are assigned as hypothetical proteins and their functions need to be further investigated.

As indicated by Figures 12 and 13, the $\Delta 12 / \omega 3$ desaturase subfamily was integrated by two main groups. Group 1 included the $\Delta 12$ desaturases from Synechococcus, Prochlorococcus and $\Delta 5$ desaturase from Bacillus subtilis. In Group 2, the $\Delta 12$ desaturases of cyanobacteria and the chloroplastic $\Delta 12$ desaturases of green algae, higher plants were in the basal position, leading to Cluster 1. In Cluster 2, the microsomal $\Delta 12$ desaturases of fungi, green algae, and higher plants set apart from $\Delta 12$ desaturases of Synechococcus, Prochlorococcus, Cyanidioschyzon merolae, Ostreococcus, Thalassiosira pseudonana, and Phaeodactylum tricorutum. Cluster 3 included the $\omega 3$ desaturases of cyanobacteria at the basal position, $\omega 3$ desaturases of green algae and both microsomal 


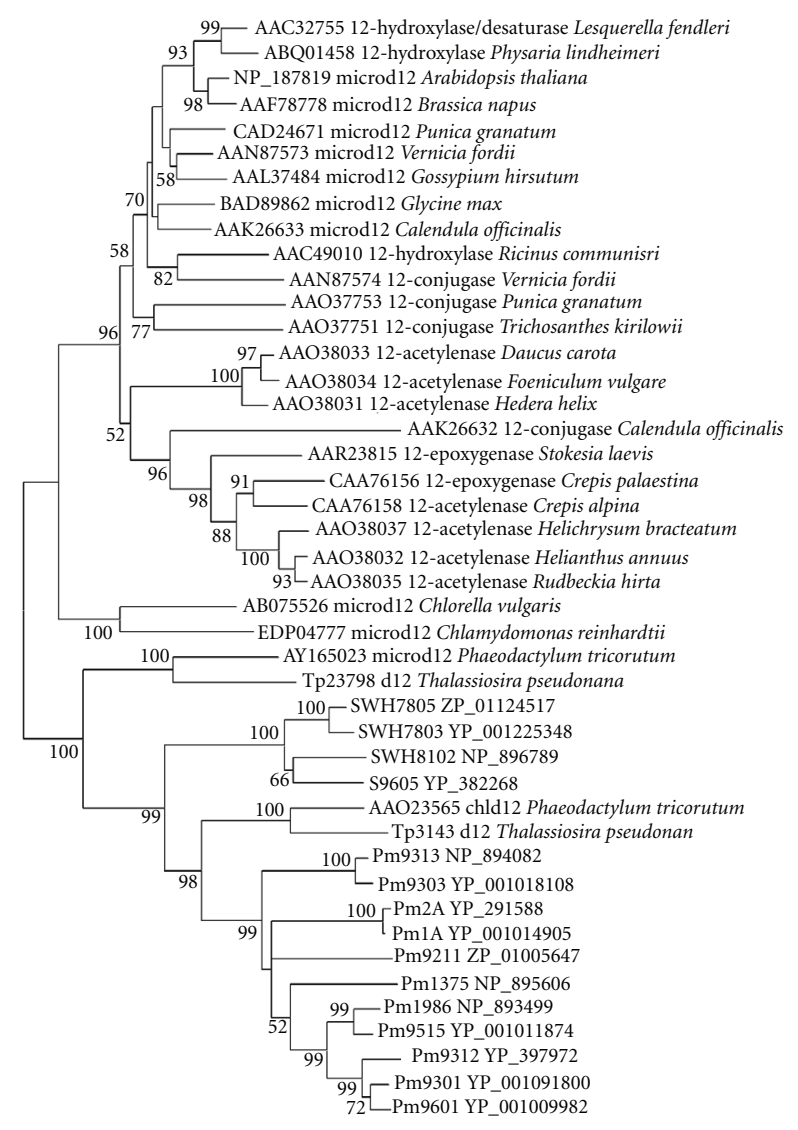

FIGURE 11: Minimum-evolution tree of $\Delta 12$ homologous genes of cyanobacteria, eukaryotic algae, and higher plants. About 300 positions spanning the three histidine-boxes were employed. Sequences from 37 sequenced cyanobacterial genomes are shown by their acronyms and accession numbers (locus tags). Other sequences are shown by their accession numbers, labels, and strain names. Desaturase genes that have been functionally characterized are indicated on the tree by their labels. Bootstrap values from minimum-evolution analyses are listed to the left of each node, with values more than 50 are shown.

and chloroplastic $\omega 3$ desaturases of higher plants. Thus, the plastidial $\Delta 12$ desaturases are ancestral to the $\omega 3$ and microsomal $\Delta 12$ desaturases, and the $\omega 3$ desaturase of higher plants and green algae arose by independent gene duplication events from prokaryotic $\omega 3$ desaturase [28].

\subsection{Discovery of Candidate Genes for $\Delta 6$ Desaturases}

The "front-end" desaturases $(\Delta 4, \Delta 5, \Delta 6$, and $\Delta 8$ desaturases) formed a separate clade, and their phylogeny is complicated (Figures 12 and 13). It has been speculated that front-end desaturases may have the same origin, but their precise lineages are still unclear. There were just four prokaryotic $\Delta 6$ desaturases found from cyanobacterial genomes in our analysis: Synechocystis sp. PCC 6803 (NP_441824), Cyanothece sp. CCY0110 (ZP_01727982), Lyngbya sp. PCC 8106 (ZP_01619238), Nodularia spumigena
CCY9414 (ZP_01632618), among which the function and molecular characteristics of $\Delta 6$ acyl-lipid desaturases from Synechocystis sp. PCC 6803 had been fully analyzed [13].

\subsection{Occurrence and Phyletic Distribution of Fatty Acid Desaturases in Thirty Seven Cyanobacteria}

In this study, thirty one unicellular and six filamentous cyanobacterial genomes were searched by bioinformatic approach for the putative fatty acid desaturases involved in polyunsaturated fatty acid synthesis. 193 protein sequences were obtained from the 37 cyanobacterial genomes, 120 of which were annotated as fatty acid desaturase. The pathway of acyl-lipid desaturation and the distribution of desaturases among different cyanobacterial species were speculated and summarized in Figures 14 and 15. Among these cyanobacteria, the $\Delta 9$ desaturase existed in 37 species of cyanobacteria. The $\Delta 12, \Delta 15$ and $\Delta 6$ desaturases existed in 31,9 , and 4 species of cyanobacteria, respectively. Based on functional criteria and the position of the clade integrated by $\Delta 9$ desaturases, $\Delta 9$ desaturase is assumed to be the ancestor of the remaining desaturases [28]. The functions performed by the latter three desaturases could have been obtained in some organisms along the evolutionary lineages.

Twenty seven of the investigated cyanobacteria come from the marine environment. These are 11 unicellular Prochlorococcus strains, 11 unicellular marine Synechococcus strains, Cyanothece sp. CCY0110, Crocosphaera watsonii WH 8501, Trichodesmium erythraeum IMS101, Lyngbya sp. PCC 8106, and Nodularia spumigena CCY9414. The other strains are from freshwater, soil, rock, hot spring, or symbiont.

In the 16S rRNA tree, marine Synechococcus and Prochlorococcus make a monophyletic group supported by a comparatively high-statistical confidence value, 100\% (Figure 1). The two genera are proposed to diverge from a common phycobilisome-containing ancestor. While marine Synechococcus still uses phycobilisomes as light-harvesting antennae, members of the Prochlorococcus genus lack phycobilisomes and use a different antenna complex that possesses derivatives of chlorophyll $a$ and $b$. They are the dominant picophytoplankton in the world's open oceans. Carbon fixation is dominated by them and together they have been shown to contribute between 32 and $80 \%$ of the primary production in oligotrophic oceans [29-32]. Synechococcus are distributed ubiquitously throughout oceanic regions, ranging from polar through temperate to tropical waters and are generally more abundant in nutrient-rich surface waters than oligotrophic areas, whilst Prochlorococcus are largely confined to a $40^{\circ} \mathrm{N} \sim 40^{\circ} \mathrm{S}$ latitudinal band, being generally absent from brackish or well-mixed waters. Prochlorococcus also generally extend deeper in the water column than Synechococcus $[33,34]$.

Prochlorococcus have been divided into two genetically and physiologically distinct groups: high- and low-B/A ecotypes, which were originally named for their difference in optimal growth irradiance (low- and high-light adapted, 


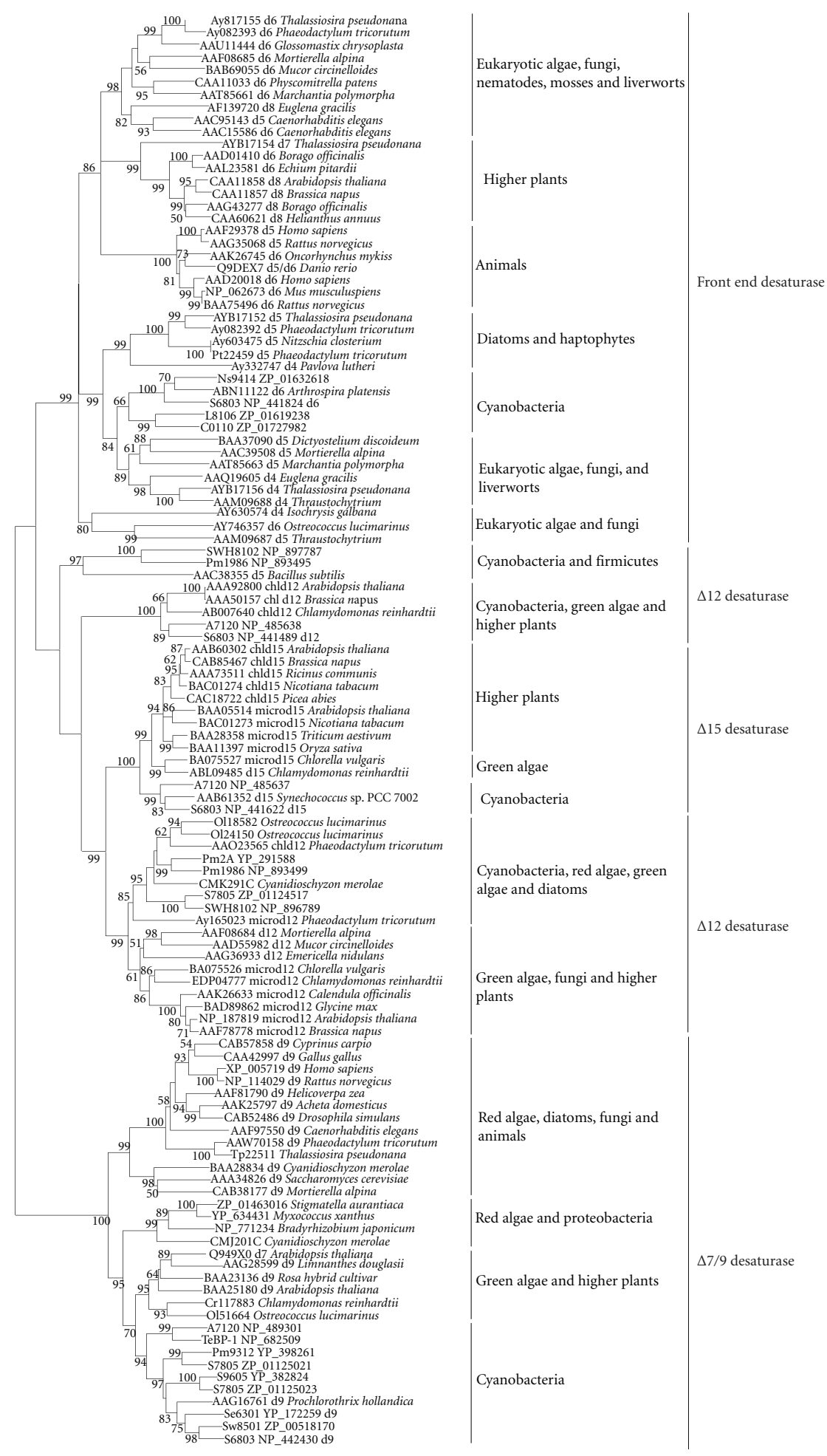

FIGURE 12: Neighbor-joining tree of membrane desaturases. About 330 positions spanning the three histidine-boxes were employed. Sequences from 37 sequenced cyanobacterial genomes are shown by their acronyms and accession numbers (locus tags). Other sequences are shown by their accession numbers, labels, and strain names. Desaturase genes that have been functionally characterized are indicated on the tree by their labels. Bootstrap values from neighbor-joining analyses are listed to the left of each node, with values more than 50 are shown. 


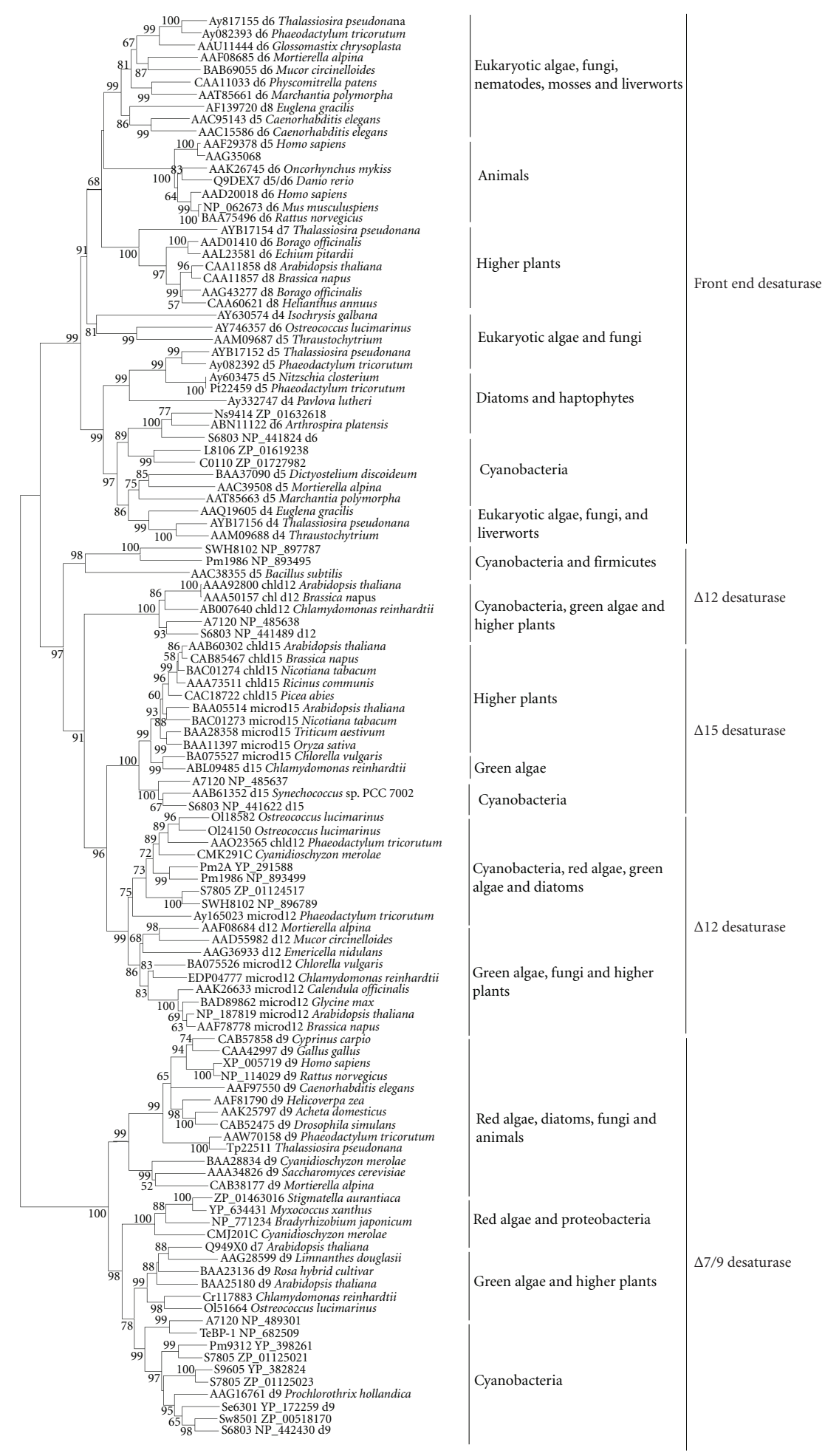

Figure 13: Minimum-evolution tree of membrane desaturases. About 330 positions spanning the three histidine-boxes were employed. Sequences from 37 sequenced cyanobacterial genomes are shown by their acronyms and accession numbers (locus tags). Other sequences are shown by their accession numbers, labels, and strain names. Desaturase genes that have been functionally characterized are indicated on the tree by their labels. Bootstrap values from minimum-evolution analyses are listed to the left of each node, with values more than 50 are shown. 


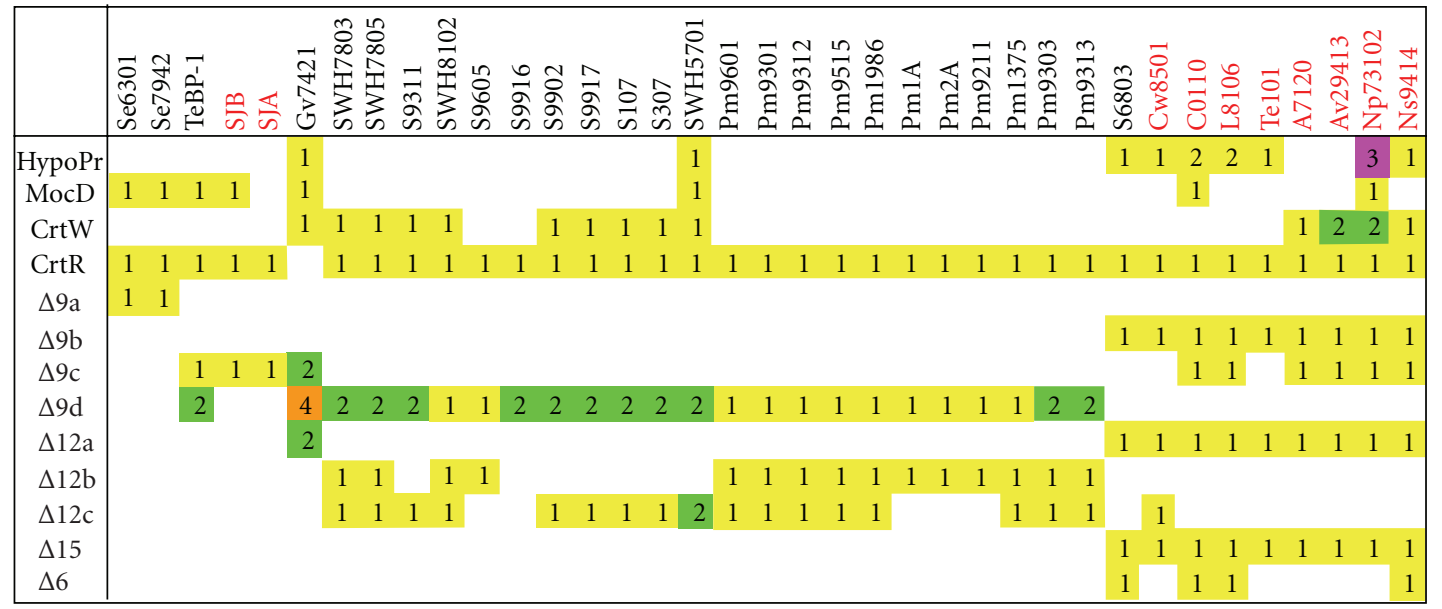

Figure 14: Diversity of different enzymes in thirty seven cyanobacteria. Distributions and amounts of different enzymes are marked by colors. One: red, two: green, three: magenta, four: orange. Names of nitrogen-fixing strains are marked in red. "HypoPr" represents hypothetical protein.

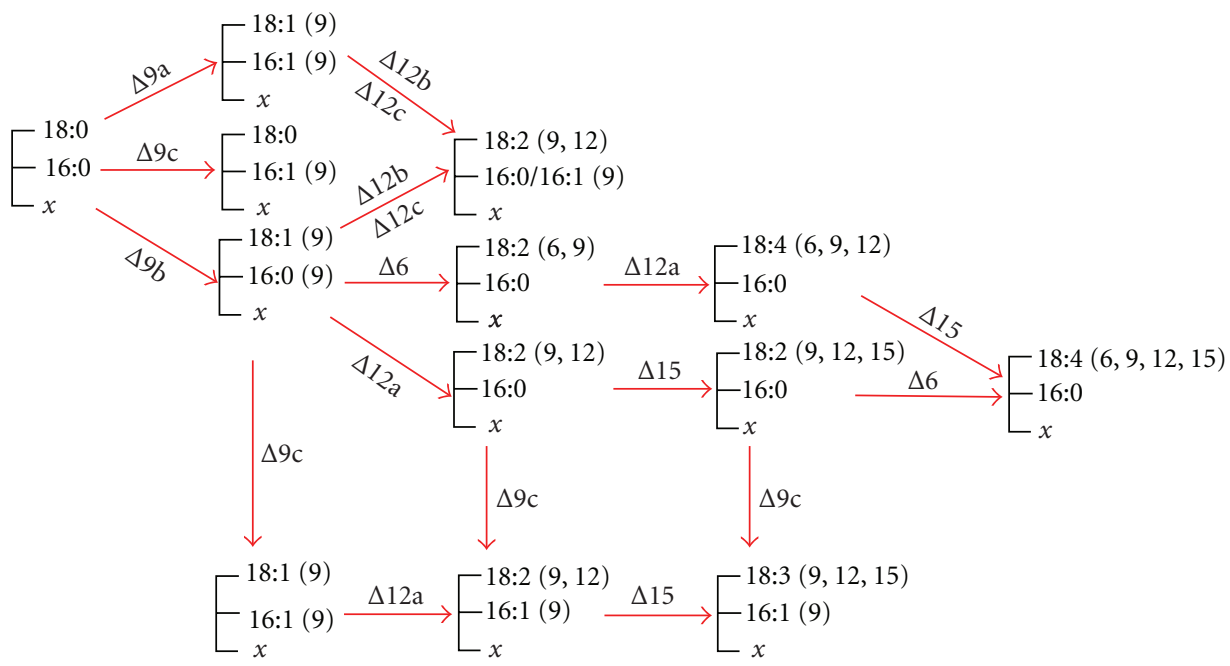

FIGURE 15: The acyl-lipid desaturation of fatty acids in cyanobacteria. Numbers around arrowhead indicate the positions at which a double bond is introduced. $\Delta 9 \mathrm{a}$ : desaturation occurring on both the $s n-1$ and the $s n-2$ positions of glycerolipids, $\Delta 9 \mathrm{~b}$ : desaturation occurring on the $s n-1$ position of glycerolipids, $\Delta 9 \mathrm{c}$ : desaturation occurring on the $s n-2$ position of glycerolipids, $\Delta 9 \mathrm{~d}$ : genes with desaturation $s n$-position of glycerolipids unspecified. $\Delta 12 \mathrm{a}$ : Clade 3 of $\Delta 12$ homologous genes, $\Delta 12 \mathrm{~b}$ : Clade 1 of $\Delta 12$ homologous genes, $\Delta 12 \mathrm{c}$ : Clade 4 of $\Delta 12$ homologous genes.

resp.) $[35,36]$. High-B/A isolates, with larger ratios of chl $b / a_{2}$, are able to grow at extremely low irradiances (less than 10 umol of quanta $[\mathrm{Q}] \mathrm{m}^{-2} \mathrm{~s}^{-1}$ ) and preferentially thrive at the bottom of the euphotic zone $(80-200 \mathrm{~m})$ at dimmer light but in a nutrient-rich environment $[37,38]$. Low-B/A isolates, have lower chl $b / a_{2}$ ratios, are able to grow maximally at higher light intensities, and occupy the upper, well illuminated but nutrient-poor 100-m layer of the water column $[37,38]$. In the $16 \mathrm{~S}$ rRNA tree, high-lightadapted Prochlorococcus sp. arises from a low-light-adapted clade (Figure 1). Prochlorococcus marinus strains AS9601, MIT 9312, MIT 9301, MIT 9515, and CCMP1986 belong to low-B/A ecotype. Their genome sizes vary from $1.6 \mathrm{Mb}$ to $1.7 \mathrm{Mb}$, smaller than that of the low light-adapted strains
(1.7 Mb to 2.6 Mb). They all contain two types of desaturases, one $\Delta 9$ desaturases and two $\Delta 12$ desaturases (b-type and c-type). Strains NATL1A, NATL2A, MIT 9211, CCMP1375, MIT 9303, and MIT 9313 belong to high-B/A ecotype. Only b-type $\Delta 12$ desaturase exists in strain NATL1A, NATL2A, and MIT 9211; while two $\Delta 9$ desaturases exist in strain MIT 9303 and MIT 9313, which have larger genome size (2.6 Mb and $2.4 \mathrm{Mb}$ ) compared to other high-B/A ecotypes.

The marine Synechococcus isolates have themselves been classified into three groups, designated marine cluster -A, -B, and -C (MC-A, MC-B, MC-C), based on the composition of the major light harvesting pigments, an ability to perform a novel swimming motility, whether they have an elevated salt requirement for growth, and $\mathrm{G}+\mathrm{C}$ content [39]. The marine 
cluster A group (mol\% G+C = 55-62), phycoerythrincontaining strains, has an elevated salt $\left(\mathrm{Na}^{+}, \mathrm{Cl}^{-}, \mathrm{Mg}^{2+}\right.$ and $\mathrm{Ca}^{2+}$ ) requirement for growth and occur abundantly within the euphotic zone of both open-ocean and coastal waters [40-44]. This cluster is additionally diverse in that ratios of phycourobilin to phycoerythrobilin chromophores differ among phycoerythrins of different strains $[45,46]$. The marine cluster B ( $\mathrm{mol} \% \mathrm{G}+\mathrm{C}=63-69.5)$ includes halotolerant strains that possess phycocyanin but lack phycoerythrin and appear confined to coastal waters. A further cluster, marine cluster $\mathrm{C}$ (MC-C) has been distinguished by its low \% G+C (47.5-49.5) containing strains from brackish or coastal marine waters [39]. These latter environments have been relatively poorly studied so far and are likely underrepresented in cultured Synechococcus isolates [33]. The b-type $\Delta 12$ desaturase only exists in strains WH 7803, WH 7805, WH 8102, and CC9605. Except for strains RS9916 and CC9605, other strains all contain c-type $\Delta 12$ desaturase, two copies of which exist in strain WH 5701 (MC-B) whose genome $(30 \mathrm{Mb})$ is larger than other Synechococcus strains $(22 \mathrm{Mb}-26 \mathrm{Mb})$. The unique characteristics can be observed in strain RS9916 that contains only $\Delta 9$ fatty acid desaturase.

The pathway of acyl-lipid desaturation for marine cyanobacteria Prochlorococcus and Synechococcus differs obviously from that of other cyanobacteria, indicating the different phylogenetic histories of the two genera from other cyanobacteria. At present, few fatty acid composition of these unicellular cyanobacteria has been determined yet, as functionally characterized genes. Therefore, the analysis on fatty acids in these cyanobacteria should provide more meaningful information for further research.

The two closely related freshwater Synechococcus elongatus strains PCC 6301 and PCC 7942 branch outside the marine picophytoplankton group (Figure 1), which suggests that marine cyanobacteria may diverge from the freshwater cyanobacterial ancestor. The gene arrangement and nucleotide sequence of Synechococcus elongatus PCC 6301 are nearly identical to those of Synechococcus elongatus PCC 7942, except for the presence of a $188.6 \mathrm{~kb}$ inversion. Genome-wide screening only recognizes one a-type $\Delta 9$ desaturase in these two strains.

Three thermophilic unicellular strains, Thermosynechococcus elongatus BP-1 and two Synechococcus Yellowstone species, are most closely related to Gloeobacter violaceus sp. PCC 7421, and phylogenetically distinct from other cyanobacterial lineages (Figure 1). They were all isolated from the hot spring. Additionally, the latter two thermophilic strains are capable of $\mathrm{N}_{2}$ fixation with a diurnal rhythm. Genes for three types of fatty acid desaturases (desA, $\operatorname{des} B$, and $\operatorname{des} D$ ) are missing in contrast with mesophilic Synechocystis, although the fourth type $(\operatorname{des} C)$ is found in Synechococcus and Thermosynechococcus elongtus. This agrees with the absence of highly unsaturated fatty acids in lipids, which are popular in many thermophiles [47]. Synechococcus sp. JA-2-3B' $\mathrm{a}(2-13)$ as well as JA-3-3Ab contains one ctype $\Delta 9$ desaturase, whereas Thermosynechococcus elongtus contains three copies, one c-type and two unspecified types. At lower temperatures, cyanobacteria desaturate the fatty acids of membrane lipids to compensate for the decrease in membrane fluidity [48]. While at higher temperatures, the membrane fluidity increased, it is unnecessary to desaturate the fatty acids of membrane lipids to produce more unsaturated fatty acids. So the thermophilic strains lack highly unsaturated fatty acids in lipids and contain only one $\Delta 9$ desaturase in contrast with mesophilic strains, which probably due to their thermic habitats.

Gloeobacter violaceus sp. PCC 7421 was originally isolated from calcareous rock in Switzerland [49, 50]. It is an unusual unicellular cyanobacterium for the absence of thylakoid membranes, and its phycobilisomes and photosystem reaction centers are localized in the plasma membrane $[51,52]$. It is also remarkable that Sulfoquinovosyl diacylglycerol (SQDG), which is thought to have an important role in photosystem stabilization, is absent in Gloeobacter while the content of polyunsaturated fatty acids (PUFA) is high [53]. The data of the fatty acid composition of Gloeobacter violaceus are few in number and contradictory. In one case, linoleic and $\alpha$-linolenic acids were found [53]. In other work, linoleic and $\gamma$-linolenic acids were identified [54]. The occurrence of $\alpha$-linolenic or $\gamma$-linolenic acid confirms that there must be a gene in the strain that is functionally similar to the $\omega 3$ desaturase or $\Delta 6$ desaturase. Two types of desaturases, six $\Delta 9$ desaturases (two c-types and four unspecified types) and two $\Delta 12$ desaturases (atype), were recognized from this strain. One hypothetical protein (NP_923117) was also found, but the three histidinemotifs of it (HDAGH, HNQLHH, HTAHH) did not agree with the standards for a front-end or $\omega 3$ desaturase. It is this protein or another protein that performs the same function as the front-end or $\omega 3$ desaturase, which need further investigation. The types and amounts of desaturases in Gloeobacter violaceus sp. PCC 7421 are distinct to those of other cyanobacteria (Figure 14). This result may accord with the conclusion that this organism is one of the earliest ones that diverged from the cyanobacterial line [55].

Nine of the 37 cyanobacteria studied here are known to fix nitrogen (Figure 1). Four Nostocales, Nostoc punctiforme ATCC 29133, Anabaena sp. PCC 7120, Anabaena variabilis ATCC 29413, and Nodularia spumigena CCY9414, are heterocyst-forming filamentous diazotroph; the other five are nonheterocystous nitrogen fixers, which are filamentous strains Trichodesmium erythraeum IMS101, Lyngbya sp. PCC 8106, unicellular strains Crocosphaera watsonii WH 8501, Cyanothece sp. CCY0110 along with thermophic Synechococcus strains JA-2-3B' a (2-13) and JA-3-3Ab.

The diazotrophic filamentous cyanobacteria, which can form terminally differentiated, nondividing heterocysts in response to nitrogen deprivation and the ensuing intracellular accumulation of 2-oxoglutarate [56], have almost the largest genome sizes $(53 \mathrm{Mb}-90 \mathrm{Mb})$ and are isolated from soil (Anabaena PCC7120), from fresh water (Anabaena variabilis ATCC 29413), from a plant-cyanobacterial symbionsis (Nostoc punctiforme PCC73102), or from the surface of Baltic sea (Nodularia spumigena CCY9414). Three types of desaturases $(\Delta 9, \Delta 12$, and $\Delta 15)$ exist in Anabaena sp. PCC 7120, Anabaena variabilis ATCC 29413, and Nostoc punctiforme ATCC 29133, with the exception that Nodularia spumigena CCY9414 contains four types of desaturases 
$(\Delta 9, \Delta 12, \Delta 15$, and $\Delta 6)$. Moreover, phylogenetic analysis shows that the desaturase genes of the same type all cluster together for these four strains, indicating a recent common ancestor for Anabaena and Nostoc [57].

Trichodesmium erythraeum IMS101 and Lyngbya sp. PCC 8106, which belong to the Oscillatoriales, both fix $\mathrm{N}_{2}$ and do not form heterocysts (Figure 1). Trichodesmium, but not Lyngbya, is known to fix nitrogen in differentiated cells called diazocytes. Like heterocysts, diazocytes are the exclusive carriers of nitrogenase and fix nitrogen aerobically in the light, and show morphological and physiological changes [58].

Unicellular strains Crocosphaera watsonii WH 8501, Cyanothece sp. CCY0110, and Synechocystis sp. PCC 6803 belong to the Chroococcaces (Figure 1), among which the former two strains fix nitrogen presumably at night while growing photosynthetically during the day. Three types of desaturases $(\Delta 9, \Delta 12$, and $\Delta 15)$ exist in Crocosphaera watsonii WH 8501 and Trichodesmium erythraeum, while four types of desaturases $(\Delta 9, \Delta 12, \Delta 15$, and $\Delta 6)$ exist in Lyngbya sp. PCC 8106, Cyanothece sp. CCY0110 and Synechocystis sp. PCC 6803. It is worth noting that the c-type $\Delta 12$ desaturase is identified exclusively in Crocosphaera watsonii WH 8501, which may be due to horizontal gene transfer (HGT) from marine cyanobacteria Prochlorococcus and Synechococcus.

In conclusion, the filamentous or $\mathrm{N}_{2}$-fixing cyanobacteria usually possess more types of fatty acid desaturases than unicellular species. The main role of fatty acid desaturase of cyanobacteria is to modulate the fluidity of membranes, which helps to improve tolerance to physiological stressors such as low temperature, high light-induced photoinhibition, salt-induced damage, or desiccation. Thus, the amounts and types of fatty acid desaturases are various among different cyanobacterial species. This evolution scheme might have formed under the force adapting to distinct environments.

\section{Acknowledgments}

This work was supported by the Key Innovative Project of Chinese Academy of Science (KZCX2-YW-209, KZCX2-YW-216), Hi-Tech Research and Development Program (2006AA090303) of China, and the CAS/SAFEA International Partnership Program for Creative Research Teams (Research and Applications of Marine Functional Genomics). Xiaoyuan Chi and Qingli Yang contributed equally to this paper.

\section{References}

[1] D. Chapman, "Phase transitions and fluidity characteristics of lipids and cell membranes," Quarterly Reviews of Biophysics, vol. 8, no. 2, pp. 185-235, 1975.

[2] S. C. Singh, R. P. Sinha, and D. P. Häder, "Role of lipids and fatty acids in stress tolerance in cyanobacteria," Acta Protozoologica, vol. 41, no. 4, pp. 297-308, 2002.

[3] D. Scanlan, "Cyanobacteria: ecology, niche adaptation and genomics," Microbiology Today, vol. 28, pp. 128-130, 2001.
[4] D. A. Bryant, Ed., The Molecular Biology of Cyanobacteria, Kluwer Academic Publishers, Dordrecht, The Netherlands, 1994.

[5] N. Sato, N. Murata, Y. Miura, and N. Ueta, "Effect of growth temperature on lipid and fatty acid compositions in the blue-green algae, Anabaena variabilis and Anacystis nidulans," Biochimica et Biophysica Acta, vol. 572, no. 1, pp. 19-28, 1979.

[6] N. Sato and N. Murata, "Studies on the temperature shift induced desaturation of fatty acids in monogalactosyl diacylglycerol in the blue-green alga (cyanobacterium), Anabaena variabilis," Plant and Cell Physiology, vol. 22, pp. 1043-1050, 1981.

[7] H. Wada and N. Murata, "Temperature-induced changes in the fatty acid composition of the cyanobacterium, Synechocystis PCC6803," Plant Physiology, vol. 92, no. 4, pp. 1062-1069, 1990.

[8] N. Murata and H. Wada, "Acyl-lipid desaturases and their importance in the tolerance and acclimatization to cold of cyanobacteria," Biochemical Journal, vol. 308, no. 1, pp. 1-8, 1995.

[9] C. D. Stubbs and A. D. Smith, "The modification of mammalian membrane polyunsaturated fatty acid composition in relation to membrane fluidity and function," Biochimica et Biophysica Acta, vol. 779, no. 1, pp. 89-137, 1984.

[10] K. P. Coolbear, C. B. Berde, and K. M. W. Keough, "Gel to liquid-crystalline phase transitions of aqueous dispersions of polyunsaturated mixed-acid phosphatidylcholines," Biochemistry, vol. 22, no. 6, pp. 1466-1473, 1983.

[11] T. Han, R. P. Sinha, and D. P. Häder, "UV-A/blue lightinduced reactivation of photosynthesis in UV-B irradiated cyanobacterium, Anabaena sp," Journal of Plant Physiology, vol. 158, no. 11, pp. 1403-1413, 2001.

[12] Y. Nishiyama, H. Yamamoto, S. I. Allakhverdiev, M. Inaba, A. Yokota, and N. Murata, "Oxidative stress inhibits the repair of photodamage to the photosynthetic machinery," EMBO Journal, vol. 20, no. 20, pp. 5587-5594, 2001.

[13] Y. Tasaka, Z. Gombos, Y. Nishiyama, et al., "Targeted mutagenesis of acyl-lipid desaturases in Synechocystis: evidence for the important roles of polyunsaturated membrane lipids in growth, respiration and photosynthesis," EMBO Journal, vol. 15, no. 23, pp. 6416-6425, 1996.

[14] Z. Gombos, E. Kanervo, N. Tsvetkova, T. Sakamoto, E.-M. Aro, and N. Murata, "Genetic enhancement of the ability to tolerate photoinhibition by introduction of unsaturated bonds into membrane glycerolipids," Plant Physiology, vol. 115, no. 2, pp. 551-559, 1997.

[15] N. Murata, H. Wada, and Z. Gombos, "Modes of fatty-acid desaturation in cyanobacteria," Plant and Cell Physiology, vol. 33, pp. 933-941, 1992.

[16] T. Kaneko, S. Sato, H. Kotani, et al., "Sequence analysis of the genome of the unicellular cyanobacterium Synechocystis sp. strain PCC6803. II. Sequence determination of the entire genome and assignment of potential protein-coding regions," DNA Research, vol. 3, no. 3, pp. 109-136, 1996.

[17] J. D. Thompson, D. G. Higgins, and T. J. Gibson, "CLUSTAL $\mathrm{W}$ : improving the sensitivity of progressive multiple sequence alignment through sequence weighting, position-specific gap penalties and weight matrix choice," Nucleic Acids Research, vol. 22, no. 22, pp. 4673-4680, 1994.

[18] K. Tamura, J. Dudley, M. Nei, and S. Kumar, "MEGA4: molecular evolutionary genetics analysis (MEGA) software version 4.0," Molecular Biology and Evolution, vol. 24, no. 8, pp. 1596-1599, 2007. 
[19] S. Chintalapati, J. S. S. Prakash, P. Gupta, et al., "A novel $\Delta^{9}$ acyl-lipid desaturase, DesC2, from cyanobacteria acts on fatty acids esterified to the sn-2 position of glycerolipids," Biochemical Journal, vol. 398, no. 2, pp. 207-214, 2006.

[20] D. J. Edwards, B. L. Marquez, L. M. Nogle, et al., "Structure and biosynthesis of the jamaicamides, new mixed polyketidepeptide neurotoxins from the marine cyanobacterium Lyngbya majuscula," Chemistry \& Biology, vol. 11, no. 6, pp. 817833,2004

[21] J. Shanklin and E. B. Cahoon, "Desaturation and related modifications of fatty acids," Annual Review of Plant Biology, vol. 49, pp. 611-641, 1998.

[22] J. A. Broadwater, E. Whittle, and J. Shanklin, "Desaturation and hydroxylation. Residues 148 and 324 of Arabidopsis FAD2, in addition to substrate chain length, exert a major influence in partitioning of catalytic specificity," Journal of Biological Chemistry, vol. 277, no. 18, pp. 15613-15620, 2002.

[23] M. Lee, M. Lenman, A. Banaś, et al., "Identification of nonheme diiron proteins that catalyze triple bond and epoxy group formation," Science, vol. 280, no. 5365, pp. 915-918, 1998.

[24] F. J. van de Loo, B. G. Fox, and C. Somerville, "Unusual fatty acids," in Lipid Metabolism in Plants, T. S. Moore, Ed., pp. 91126, CRC Press, BocaRaton, Fla, USA, 1993.

[25] J. Shanklin, C. Achim, H. Schmidt, B. G. Fox, and E. Münck, "Mössbauer studies of alkane $\omega$-hydroxylase: evidence for a diiron cluster in an integral-membrane enzyme," Proceedings of the National Academy of Sciences of the United States of America, vol. 94, no. 7, pp. 2981-2986, 1997.

[26] F. Domergue, P. Spiekermann, J. Lerchl, et al., "New insight into Phaeodactylum tricornutum fatty acid metabolism. Cloning and functional characterization of plastidial and microsomal $\Delta^{12}$-fatty acid desaturases," Plant Physiology, vol. 131, no. 4, pp. 1648-1660, 2003.

[27] P. S. Aguilar, J. E. Cronan Jr., and D. De Mendoza, "A Bacillus subtilis gene induced by cold shock encodes a membrane phospholipid desaturase," Journal of Bacteriology, vol. 180, no. 8, pp. 2194-2200, 1998.

[28] D. López Alonso, F. García-Maroto, J. Rodríguez-Ruiz, J. A. Garrido, and M. A. Vilches, "Evolution of the membranebound fatty acid desaturases," Biochemical Systematics and Ecology, vol. 31, no. 10, pp. 1111-1124, 2003.

[29] R. Goericke and N. A. Welschmeyer, "The marine prochlorophyte Prochlorococcus contributes significantly to phytoplankton biomass and primary production in the Sargasso Sea," Deep Sea Research Part I, vol. 40, no. 11-12, pp. 2283-2294, 1993.

[30] W. K. Li, "Composition of ultraphytoplankton in the central north Atlantic," Marine Ecology Progress Series, vol. 122, no. 1-3, pp. 1-8, 1995.

[31] H. Liu, H. A. Nolla, and L. Campbell, "Prochlorococcus growth rate and contribution to primary production in the equatorial and subtropical North Pacific Ocean," Aquatic Microbial Ecology, vol. 12, no. 1, pp. 39-47, 1997.

[32] M. J. W. Veldhuis, G. W. Kraay, J. D. L. Van Bleijswijk, and M. A. Baars, "Seasonal and spatial variability in phytoplankton biomass, productivity and growth in the northwestern Indian ocean: the southwest and northeast monsoon, 1992-1993," Deep Sea Research Part I, vol. 44, no. 3, pp. 425-449, 1997.

[33] D. J. Scanlan and N. J. West, "Molecular ecology of the marine cyanobacterial genera Prochlorococcus and Synechococcus," FEMS Microbiology Ecology, vol. 40, no. 1, pp. 1-12, 2002.
[34] F. Partensky, W. R. Hess, and D. Vaulot, "Prochlorococcus, a marine photosynthetic prokaryote of global significance," Microbiology and Molecular Biology Reviews, vol. 63, no. 1, pp. 106-127, 1999.

[35] G. Rocap, L. R. Moore, and S. W. Chisholm, "Molecular phylogeny of Prochlorococcus ecotypes," in Marine Cyanobacteria, L. Charpy and A. W. D. Larkum, Eds., pp. 107-116, Bulletin de l'Institut Océanographique, Monaco, France, 1999.

[36] L. R. Moore, G. Rocap, and S. W. Chisholm, "Physiology and molecular phytogeny of coexisting Prochlorococcus ecotypes," Nature, vol. 393, no. 6684, pp. 464-467, 1998.

[37] L. R. Moore and S. W. Chisholm, "Photophysiology of the marine cyanobacterium Prochlorococcus: ecotypic differences among cultured isolates," Limnology and Oceanography, vol. 44, no. 3, pp. 628-638, 1999.

[38] A. Dufresne, M. Salanoubat, F. Partensky, et al., "Genome sequence of the cyanobacterium Prochlorococcus marinus SS120, a nearly minimal oxyphototrophic genome," Proceedings of the National Academy of Sciences of the United States of America, vol. 100, no. 17, pp. 10020-10025, 2003.

[39] J. B. Waterbury and R. Rippka, "Subsection 1. Order Croococcales Wettsten 1924, emend. Rippka et al., 1979," in Bergey's Manual of Systematic Bacteriology, J. T. Staley, M. P. Bryant, N. Pfenning, and J. G. Holt, Eds., vol. 3, pp. 1728-1746, Williams and Wilkins, Baltimore, Md, USA, 1989.

[40] M. J. Ferris and B. Palenik, "Niche adaptation in ocean cyanobacteria," Nature, vol. 396, no. 6708, pp. 226-228, 1998.

[41] F. Partensky, J. Blanchot, and D. Vaulot, "Differential distribution and ecology of Prochlorococcus and Synechococcus in oceanic waters: a review," Bulletin de l'Institut Oceanographique, vol. 19, pp. 457-475, 1999.

[42] Z. Uysal, "Pigments, size and distribution of Synechococcus spp. in the Black Sea," Journal of Marine Systems, vol. 24, no. 3-4, pp. 313-326, 2000.

[43] A. Wilmotte, C. Demonceau, A. Goffart, J.-H. Hecq, V. Demoulin, and A. C. Crossley, "Molecular and pigment studies of the picophytoplankton in a region of the Southern Ocean $\left(42-54^{\circ} \mathrm{S}, 141-144^{\circ} \mathrm{E}\right)$ in March 1998," Deep Sea Research Part II, vol. 49, no. 16, pp. 3351-3363, 2002.

[44] A. M. Wood, D. A. Phinney, and C. S. Yentsch, "Water column transparency and the distribution of spectrally distinct forms of phycoerythrin-containing organisms," Marine Ecology Progress Series, vol. 162, pp. 25-31, 1998.

[45] A. M. Wood, P. K. Horan, K. Muirhead, D. A. Phinney, C. M. Yentsch, and J. B. Waterbury, "Discrimination between types of pigments in marine Synechococcus sp. by scanning spectroscopy, epifluorescence microscopy, and flow cytometry," Limnology and Oceanography, vol. 30, pp. 1303-1315, 1985.

[46] J. B. Waterbury, S. W. Watson, F. W. Valois, and D. G. Franks, "Biological and ecological characterisation of the marine unicellular cyanobacterium Synechococcus," Canadian Bulletin of Fisheries and Aquatic Sciences, vol. 214, pp. 71-120, 1986.

[47] Y. Nakamura, T. Kaneko, S. Sato, et al., "Complete genome structure of the thermophilic cyanobacterium Thermosynechococcus elongatus BP-1," DNA Research, vol. 9, no. 4, pp. 123130, 2002.

[48] N. Murata and I. Nishida, "Lipids of blue-green algae (cyanobacteria)," in The Biochemistry of Plants, P. K. Stumpf, Ed., pp. 315-347, Academic Press, San Diego, Calif, USA, 1987.

[49] R. Rippka, J. Waterbury, and G. Cohen-Bazire, "A cyanobacterium which lacks thylakoids," Archives of Microbiology, vol. 100, no. 4, pp. 419-436, 1974. 
[50] R. Rippka and M. Herdman, Pasteur Culture Collection (PCC) of Cyanobacterial Strains in Axenic Culture, vol. 1 of Catalogue of Strains, Institute Pasteur, Paris, France, 1992.

[51] Y. Nakamura, T. Kaneko, S. Sato, et al., "Complete genome structure of Gloeobacter violaceus PCC 7421, a cyanobacterium that lacks thylakoids," DNA Research, vol. 10, no. 4, pp. 137145, 2003.

[52] G. Guglielmi, G. Cohen-Bazire, and D. A. Bryant, "The structure of Gloeobacter violaceus and its phycobilisomes," Archives of Microbiology, vol. 129, no. 3, pp. 181-189, 1981.

[53] E. Selstam and D. Campbell, "Membrane lipid composition of the unusual cyanobacterium Gloeobacter violaceus sp. PCC 7421, which lacks sulfoquinovosyl diacylglycerol," Archives of Microbiology, vol. 166, no. 2, pp. 132-135, 1996.

[54] R. Rippka, J. Waterbury, and G. Cohen-Bazire, "A cyanobacterium which lacks thylakoids," Archives of Microbiology, vol. 100, no. 4, pp. 419-436, 1974.

[55] D. Honda, A. Yokota, and J. Sugiyama, "Detection of seven major evolutionary lineages in cyanobacteria based on the $16 \mathrm{~S}$ rRNA gene sequence analysis with new sequences of five marine Synechococcus strains," Journal of Molecular Evolution, vol. 48, no. 6, pp. 723-739, 1999.

[56] C.-C. Zhang, S. Laurent, S. Sakr, L. Peng, and S. Bédu, "Heterocyst differentiation and pattern formation in cyanobacteria: a chorus of signals," Molecular Microbiology, vol. 59, no. 2, pp. 367-375, 2006.

[57] R. F. McGuire, "A numerical taxonomic study of Nostoc and Anabaena," Journal of Phycology, vol. 20, pp. 454-460, 1984.

[58] R. El-Shehawy, C. Lugomela, A. Ernst, and B. Bergman, "Diurnal expression of hetR and diazocyte development in the filamentous non-heterocystous cyanobacterium Trichodesmium erythraeum," Microbiology, vol. 149, no. 5, pp. 1139-1146, 2003. 

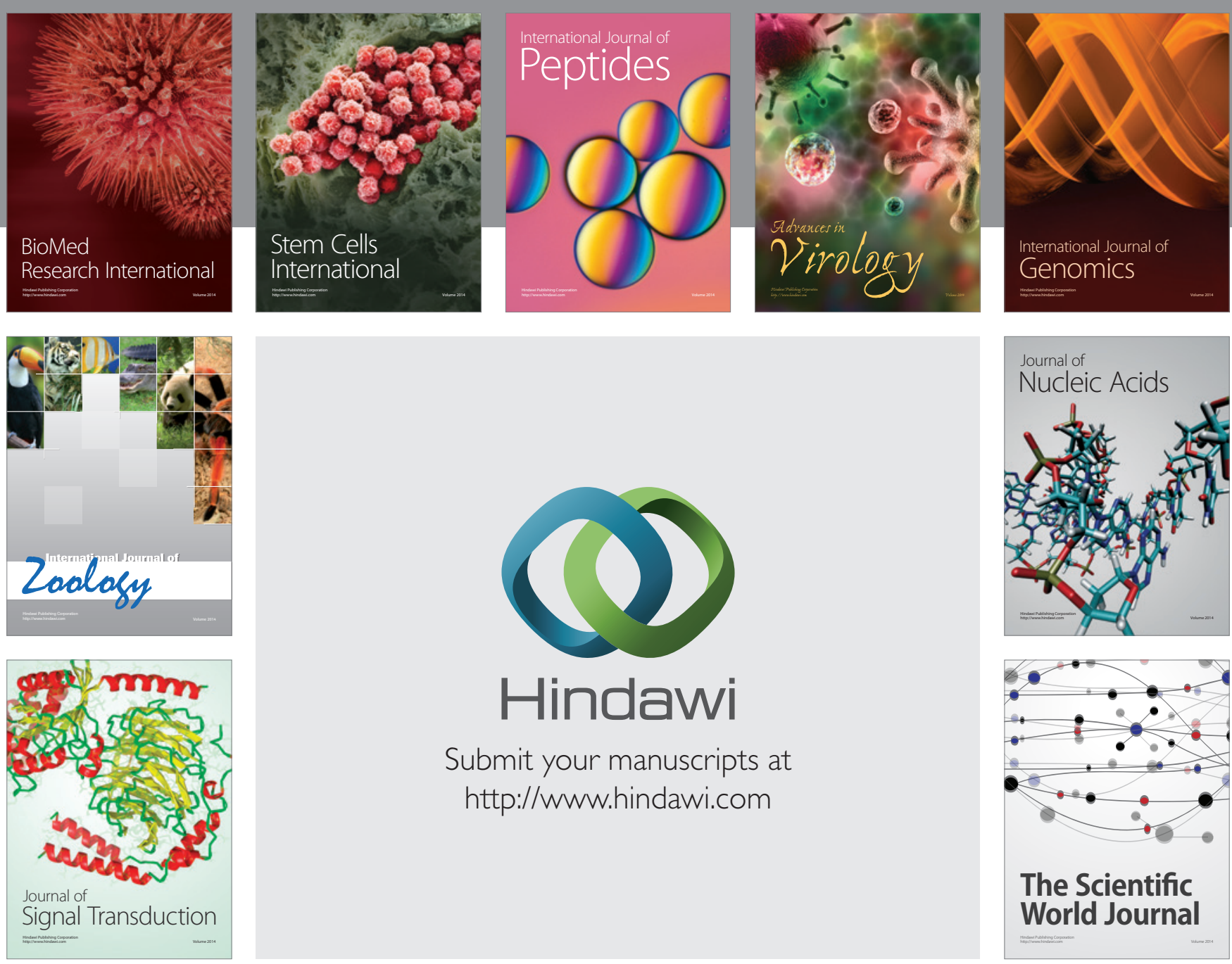

Submit your manuscripts at

http://www.hindawi.com
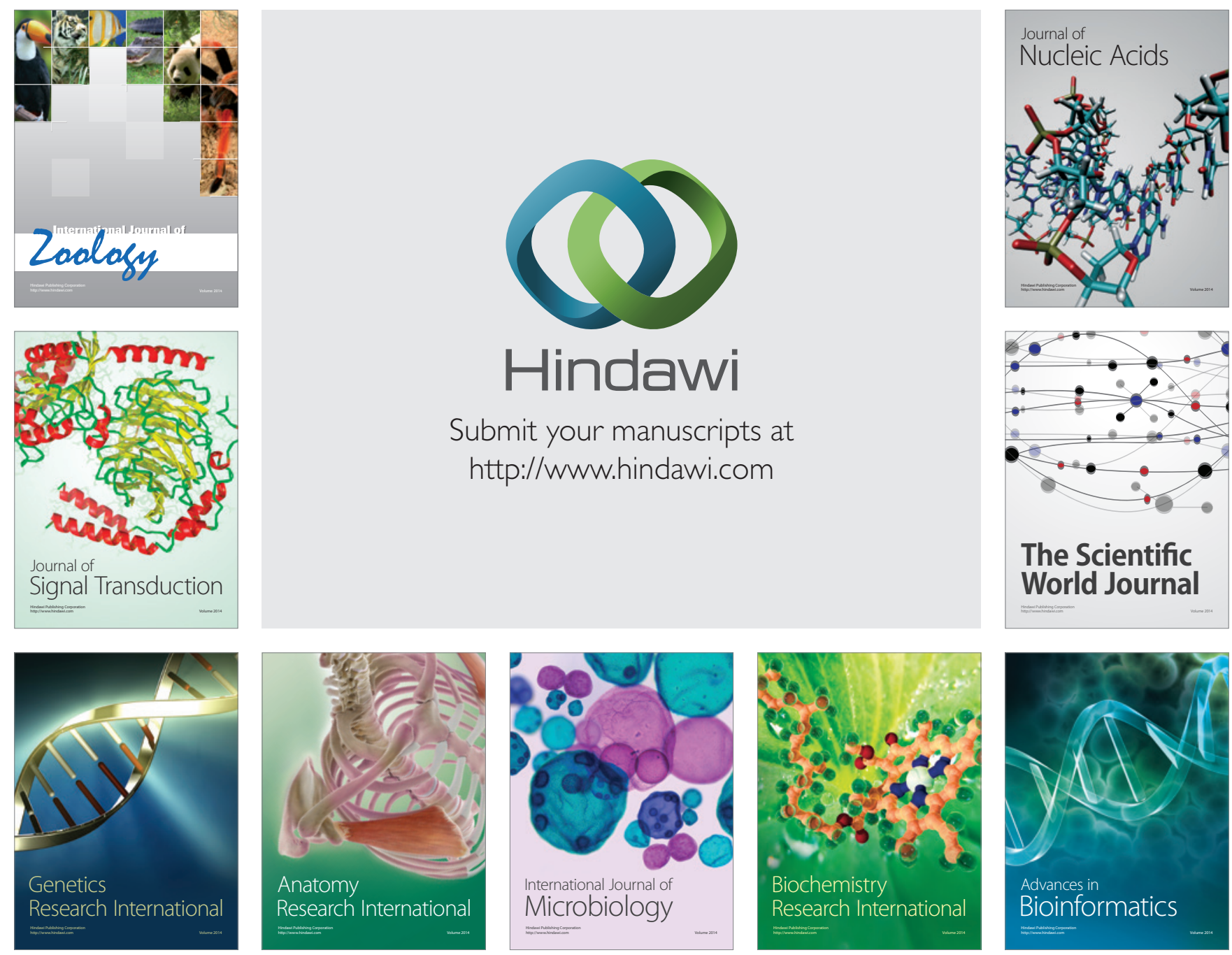

The Scientific World Journal
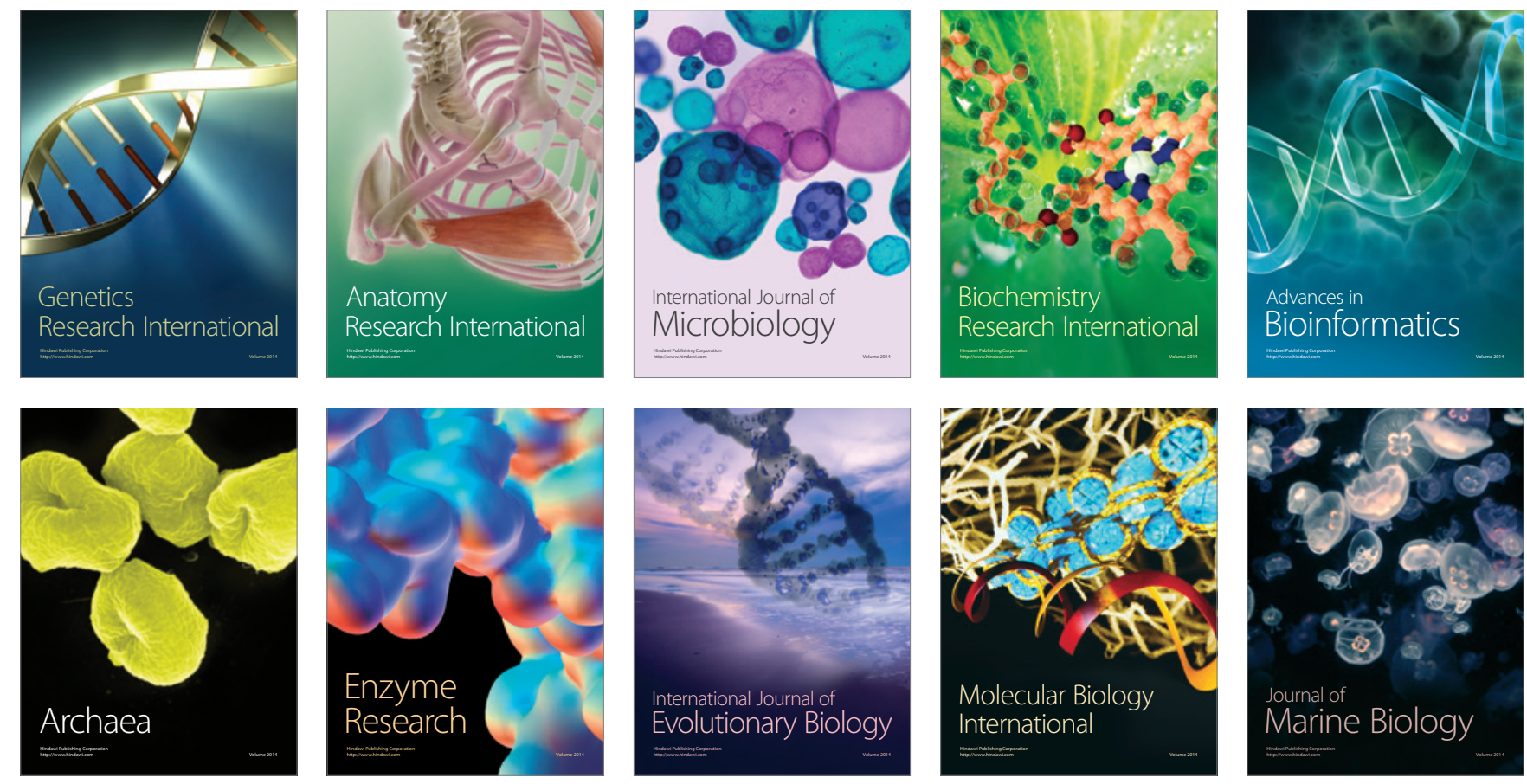